\title{
Velocity distribution for a two-dimensional sheared granular flow
}

\author{
M. Bose and V. Kumaran \\ Department of Chemical Engineering, Indian Institute of Science, Bangalore, India
}

\begin{abstract}
The velocity distribution for a two-dimensional collection of disks of number density $n$ per unit area and radius $a$ in a channel of width $L$ is studied. The particle-particle collisions are considered to be inelastic with a coefficient of restitution $e$, while the particle-wall coefficients of restitution are inelastic with a tangential and normal coefficients of restitution, $e_{\mathrm{t}}$ and $e_{\mathrm{n}}$, respectively. The Knudsen number, which is the ratio of the channel width and the mean free path of the particles, is varied from $\mathrm{Kn} \ll 1$ to $\mathrm{Kn} \gg 1$. In the limit of high Knudsen number, the distribution function for the streamwise velocity is bimodal, as predicted by theory [V. Kumaran, J. Fluid Mech., 340, 319 (1997)], and the scalings of the moments of the velocity distribution with the Knudsen number are in agreement with the theory. In the low Knudsen number limit, the distribution function for the streamwise velocity is a Gaussian if the coefficient of restitution is close to 1, but assumes the form of a "composite Gaussian" if the coefficient of restitution is not close to 1. The distribution function has a complex structure in the intermediate regime, where there are three maxima in the distribution function near the wall, while the distribution function is bimodal at the center. The granular temperature is accurately predicted by kinetic theory at the center of the channel, but there is dissipation at the wall due to inelastic particle-wall collisions, which results in a significant decrease in the temperature even when the coefficient of restitution is 0.9 ; this finding is in agreement with previous results with bumpy wall boundary conditions and with specular reflection conditions. The slip velocity at the wall has a power law dependence on the Knudsen number, and the exponent in this power law depends on the coefficients of restitution.
\end{abstract}

\section{INTRODUCTION}

The shear flow of a granular material has been an extensively studied problem in the field of granular flows, and many unusual phenomena, such as the formation of dense clusters and inhomogeneities [1], have been observed. Two distinct types of approaches have been involved in studying these systems, the continuum theories for slow flows, and kinetic theories for rapid flows. In slow flows, the particles are in extended contact with each other, and a transfer of the momentum and energy occurs due to rubbing friction, while in rapid flows momentum and energy are transported due to instantaneous collisions between the particles. The plane Couette flow of a granular material driven by the motion of parallel walls is a commonly studied example of a rapid flow. The kinetic theories for rapid flows exploit the analogy between the collisional dynamics of the molecules in a gas and that of the particles in a granular flow, and an assumption of molecular chaos is made while formulating the Boltzmann equation for the particle velocity distribution. However, the dynamics of a granular flow could be significantly different from that of a molecule of gas for a variety of reasons. The particles in a granular medium are macroscopic, and the length scale of the flow is typically of the same magnitude as the mean free path. Moreover, the collisions between the particles in a granular medium are inelastic, and do not conserve energy. Therefore, a continuous source of energy is required to sustain the flow. In a shear flow, the walls act as the source of energy, and the "granular temperature," which is a measure of the fluctuating energy of the particles, is determined from the balance between the source of energy due to the mean shear and the dissipation of energy due to inelastic collisions. In contrast to a molecular gas, the granu- lar temperature is not an equilibrium thermodynamic property. Since a net energy flux is required to sustain the motion of the particles, there could be gradients of density and temperature at steady state.

The kinetic theory description of a granular material requires a solution of the Boltzmann equation, which expresses the conservation of the number of particles in phase space. It is difficult to solve this equation, because the collisional term in the equation is nonlinear in the distribution function and nonlocal in the velocity coordinates. It can, however, be shown that, for an ideal gas in equilibrium, the solution to this equation is a Gaussian distribution. For systems near equilibrium, an asymptotic analysis can be employed with the equilibrium distribution as the leading approximation. Most previous studies on rapid shear flows have used this method, but this approach is applicable only in parameter regimes where the coefficient of restitution $e$ is close to 1 ,

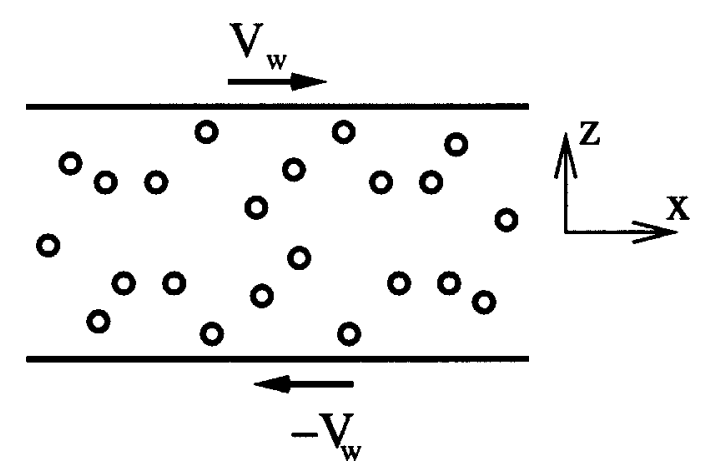

FIG. 1. Schematic of a two-dimensional bounded plane shear flow. 


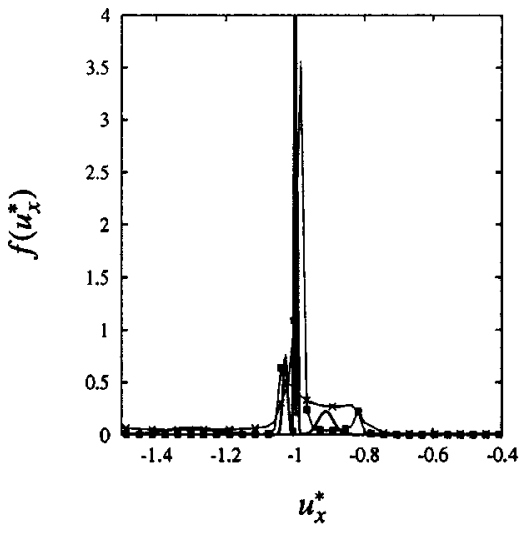

(a)

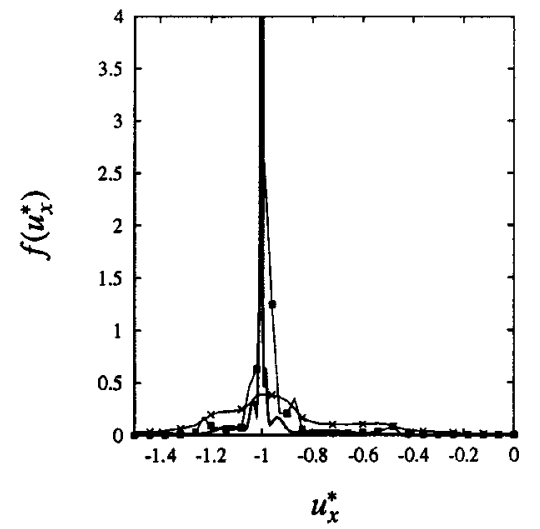

(b)

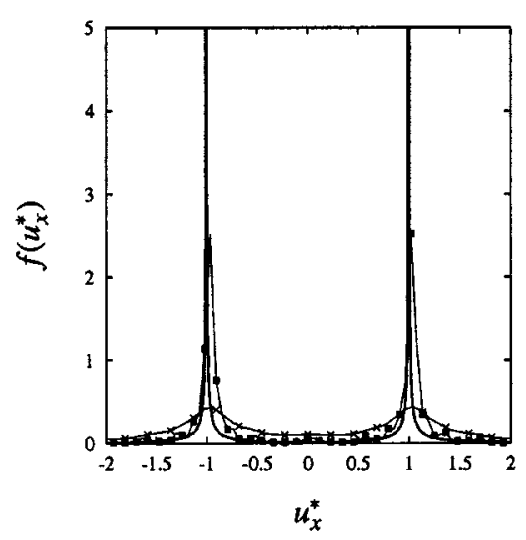

(c)

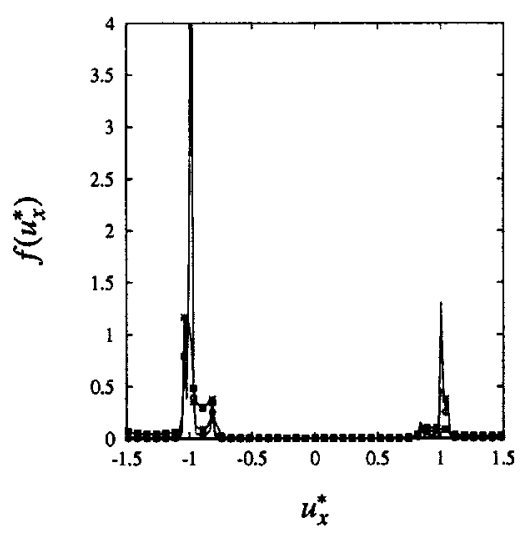

(a)

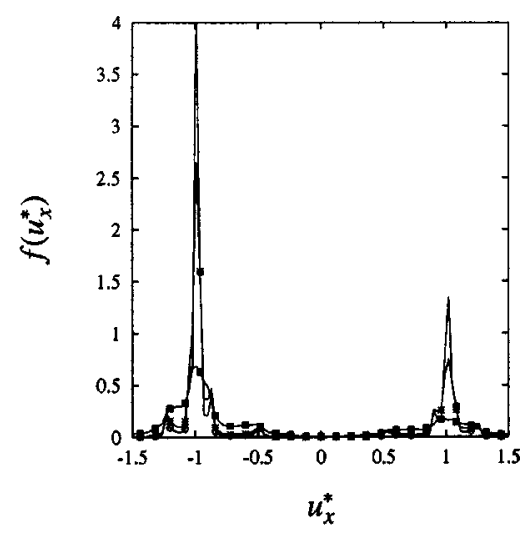

(b)

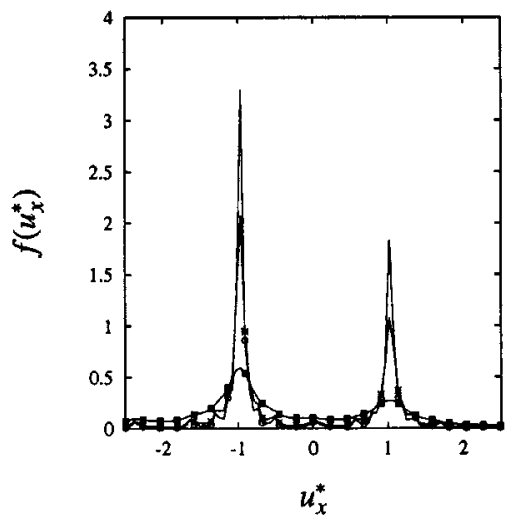

(c)
FIG. 2. Distribution function for the streamwise velocity for different coefficients of restitutions at the center of the channel. (a) $e_{\mathrm{t}}=e_{\mathrm{n}}=0.3$, (b) $e_{\mathrm{t}}=e_{\mathrm{n}}=0.5$, and (c) $e_{\mathrm{t}}=e_{\mathrm{n}}=0.7$. (回) $\epsilon=0.005$, $(\times) \epsilon=0.05$, and (-) Theory [11]. In the first two cases only one half of the distribution is shown for clarity. It is symmetric about zero velocity.

FIG. 3. Distribution function for the streamwise velocity for different coefficients of restitutions at $z / L=-0.9$ in the channel. (a) $e_{\mathrm{t}}=e_{\mathrm{n}}=0.3$, (b) $e_{\mathrm{t}}=e_{\mathrm{n}}=0.5$, and (c) $e_{\mathrm{t}}=e_{\mathrm{n}}=0.7$. (०) $\epsilon=0.005$, $(*) \epsilon=0.001$, and $(\mathbf{\square}) \epsilon=0.05$. 


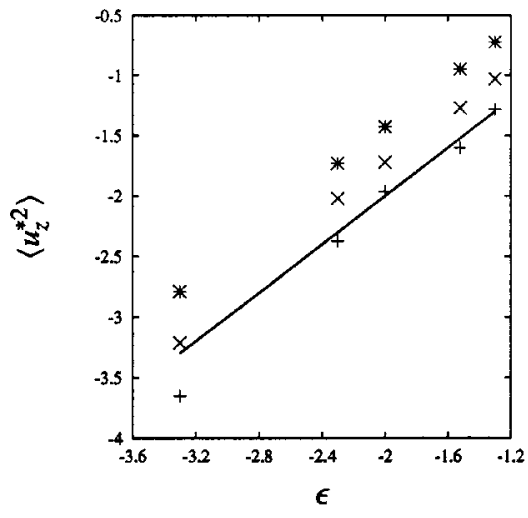

(a) (b)
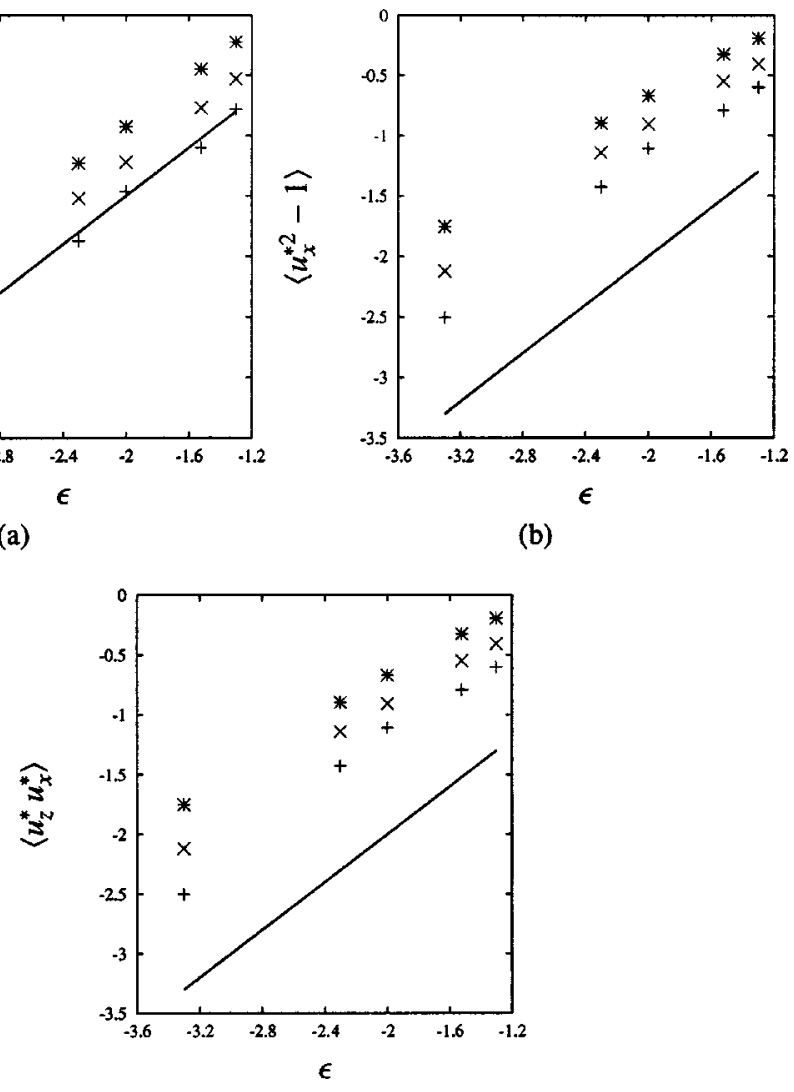

(c)

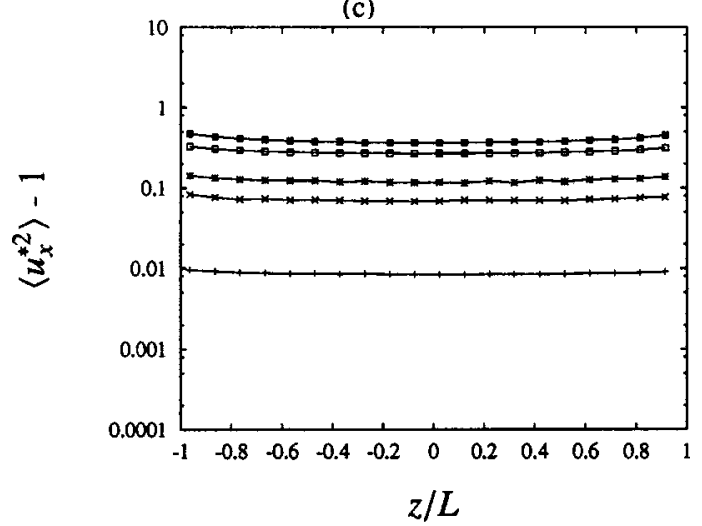

(a)

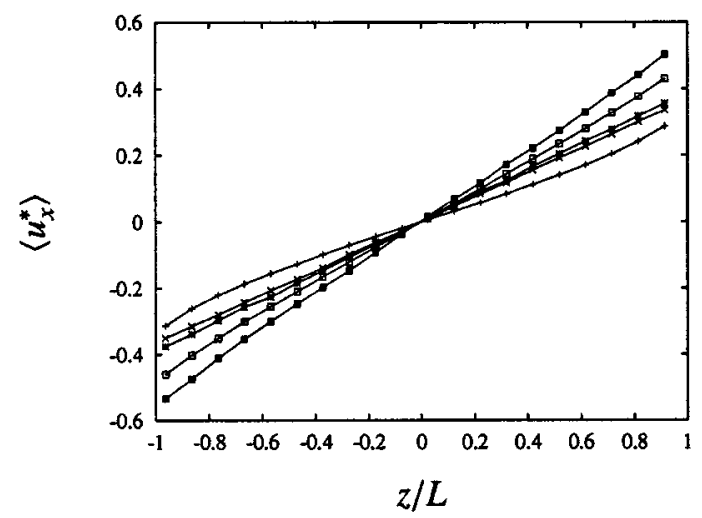

(c)
FIG. 4. Scaling of different moments of the velocity distribution function. (a) $\left\langle u_{z}^{* 2}-1\right\rangle$, (b) $\left\langle u_{x}^{* 2}-1\right\rangle$, and (c) $\left\langle u_{z}^{*} u_{x}^{*}\right\rangle$ with $\epsilon$. Points represent the results obtained from simulation for different values of $e_{\mathrm{t}}$ and $e_{\mathrm{n}},(*) e_{\mathrm{t}}=e_{\mathrm{n}}=0.7,(\times) e_{\mathrm{t}}=e_{\mathrm{n}}$ $=0.5$, and $(+) e_{\mathrm{t}}=e_{\mathrm{n}}=0.3$, and the solid line is a straight line with slope 1 .

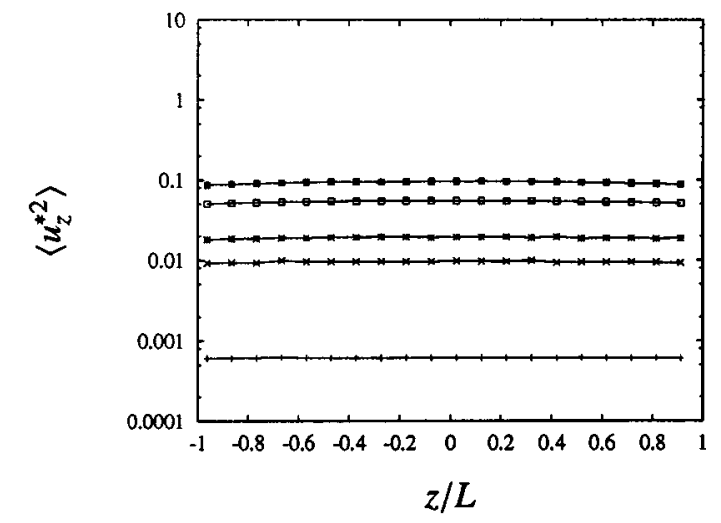

(b)

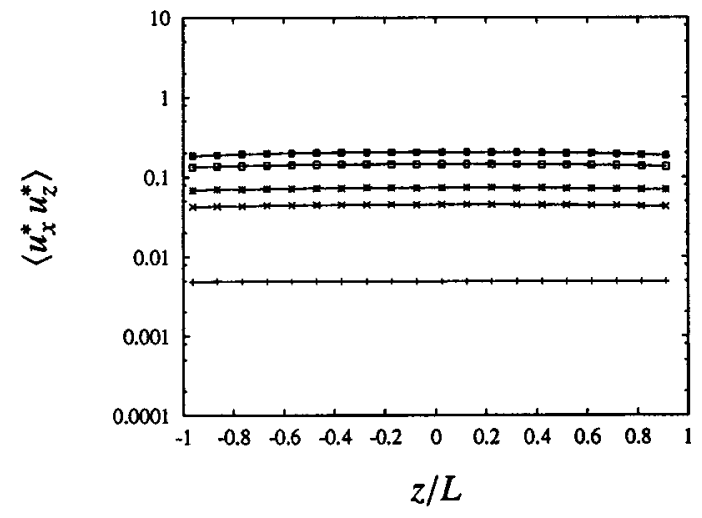

(d)

FIG. 5. Variation of the scaled velocity moments along the channel height for $e_{\mathrm{t}}=e_{\mathrm{n}}=0.5, e=1$. $(+) \epsilon=0.0005,(\times) \epsilon=0.005,(*) \epsilon$ $=0.01,(\square) \epsilon=0.03$, and (ם) $\epsilon=0.05$. 


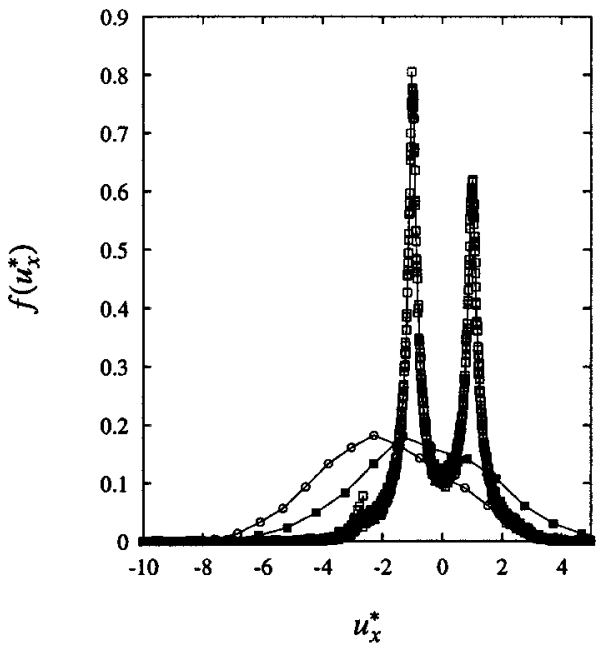

(a)

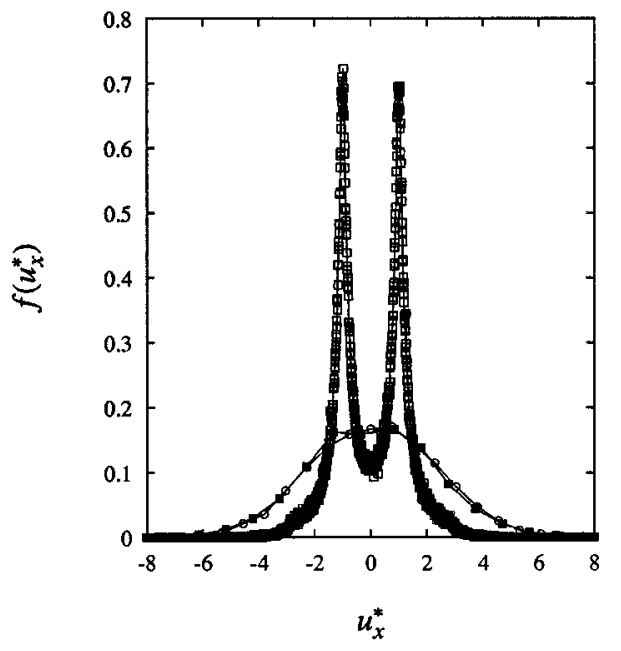

(c)

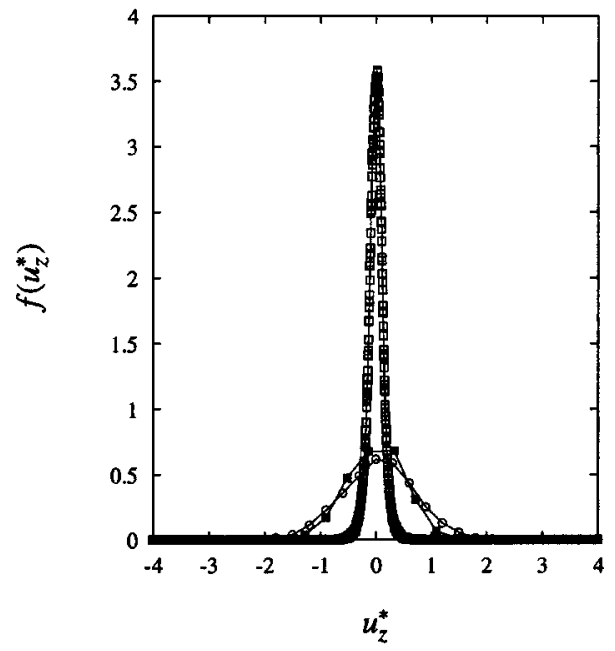

(b)

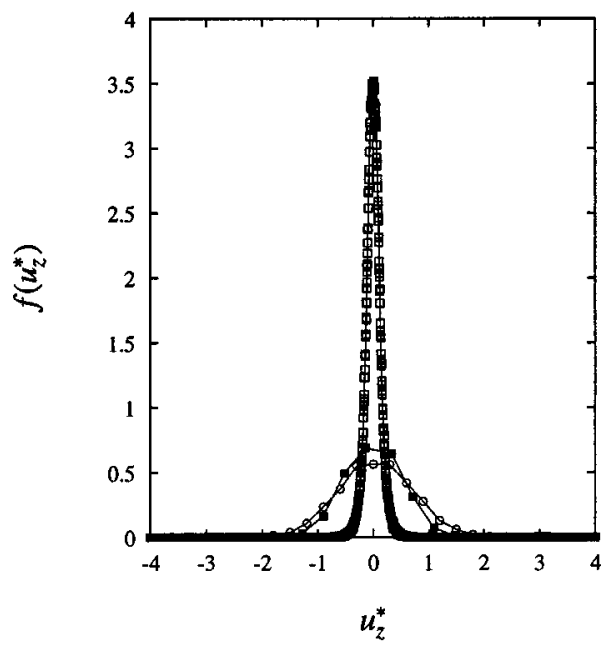

(d)

FIG. 6. Distribution of streamwise [(a) and (c)] and cross-stream [(b) and (d)] velocities near the bottom wall [(a) and (b)] and at the center [(c) and (d)] of the channel for different values of $\epsilon$ and $e=0.9$. ( $\square) \epsilon=0.01,(\square) \epsilon=0.1$, and (०) $\epsilon=1.0$.

the time between successive collisions is small compared to the inverse strain rate, and the distance between walls is large compared to the mean free path (low Knudsen number). It is of interest to determine the form of the distribution when these conditions are not satisfied, and the form of the distribution function for a specific boundary configuration is determined using event driven simulations in the present analysis.

Constitutive equations for a rapid flow of granular materials under shear, based on the Chapman-Enskog method for inelastic spheres and circular disks, were proposed by Lun et al. [2] and Jenkins and Richman [3]. Boundary conditions for a specific wall geometry, based on a detailed microscopic model for wall-particle interaction, were obtained [4,5]. A constitutive relation incorporating both the collisional and frictional transport of momentum for simpler specularity boundary conditions was proposed by Johnson and Jackson [6]. Bounded shear flows have also been studied, usually with the 'bumpy wall' boundary conditions, where the walls of the channel are decorated with hemispheres in order to effect momentum and energy transport to the particles, since smooth walls do not transfer momentum in the flow direction. The thickness required to obtain a steady flow in a channel of finite thickness has been examined by Hanes et al. [7]. Numerical simulations of flows with bumpy walls have been carried out by Lun [8], and these simulations incorporated the tangential and rotational velocities of the particles. Particular attention was paid to the slip velocity at the walls for different arrangements of the hemispheres on the wall surface. Babic [9] examined unsteady startup and oscillatory flows using discrete element method simulations, and found that the granular material behaves as a nearly incompressible non-Newtonian fluid when the energy relaxation time is long compared to the momentum diffusion time. All of the above approaches have used continuum theories, which are valid in the limit of a low Knudsen number, where the frequency of binary collisions is large compared to that of particle-wall collisions. In addition, balance equations are only written for the hydrodynamic mass, momentum, and energy fields, and not the velocity distribution function itself. The velocity distribution function in a channel was evaluated by Tij et al. [10] using the linearized Bhatnagar-Gross-Krook equation. 


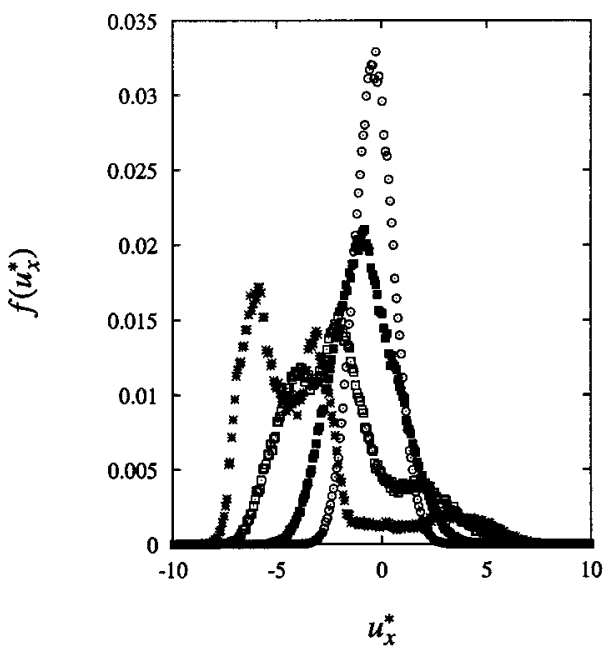

(a)

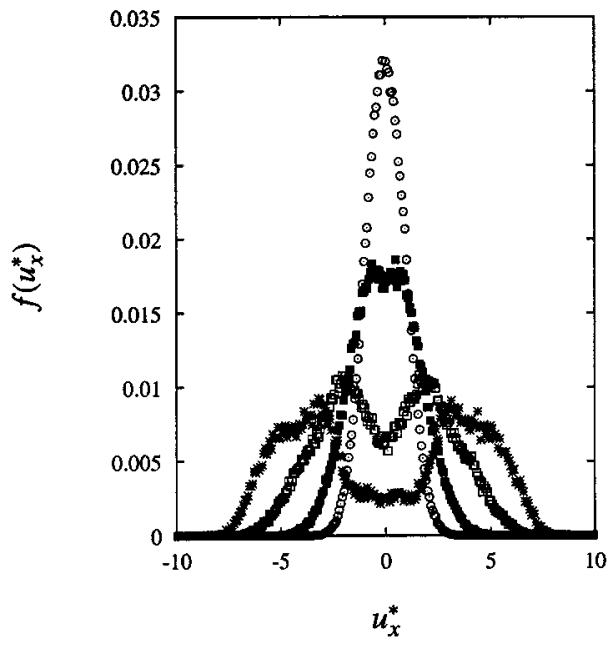

(c)

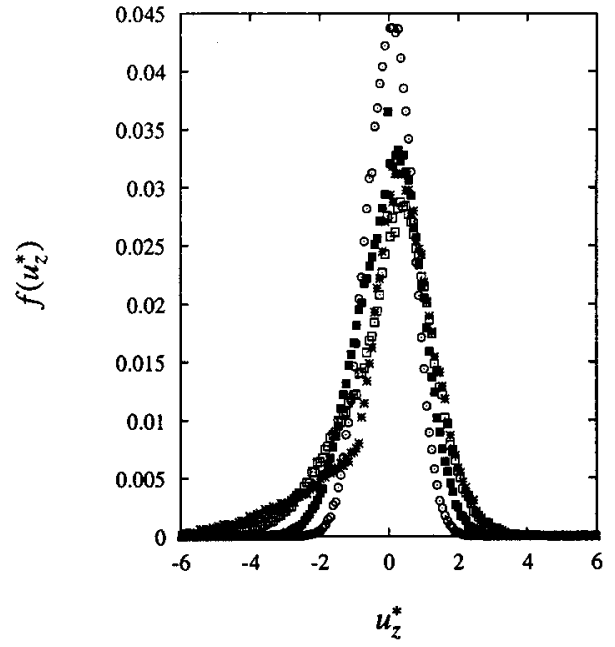

(b)

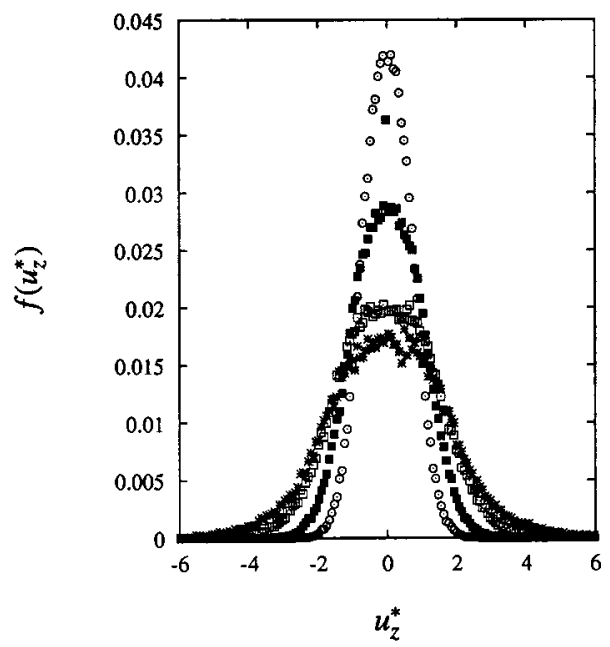

(d)

FIG. 7. Distribution of streamwise [(a) and (c)] and cross-stream [(b) and (d)] velocities near the bottom wall [(a) and (b)] and at the center (c) and (d)] of the channel for different values of $e$ and $\epsilon=0.3$. (*) $e=0.4,(\square) e=0.6,(\square) e=0.8$, and (o) $e=0.9$.

The linearization approximation assumed a collision relaxation to a Maxwell-Boltzmann distribution, and this considerably simplified the calculation procedure. In addition, idealized boundary conditions, such as the zero temperature conditions at the wall, were assumed. This calculation was also carried out in the low Knudsen number limit, and boundary layers were observed for the hydrodynamic fields near the walls where there were sharp variations in the temperature.

The above theories usually assume that the distribution function is close to a Maxwell-Boltzmann distribution, or is collisionally relaxed to a Maxwell-Boltzmann distribution. This assumption is valid only when the coefficient of restitution is close to 1 , and in the low Knudsen number limit where the mean free path is small compared to the channel width. In contrast, the velocity distribution function was determined analytically [11] in the high Knudsen number limit, where the mean free path is of the same magnitude as the channel width, using an asymptotic analysis in the small parameter $\epsilon$, where $\epsilon=n \sigma L, n$ is the number density, $\sigma$ is the diameter of the particles, and $L$ is the channel width. The particle-wall collisions are considered to be inelastic and are described by simple relations which include coefficients of restitution in the tangential and normal directions $\left(e_{\mathrm{t}}\right.$ and $\left.e_{\mathrm{n}}\right)$, respectively. Both elastic and inelastic binary collisions between particles were considered. This study indicated that the distribution function is very different from a MaxwellBoltzmann distribution in this limit. In the present study, the predictions of this analysis are compared to the results of event driven simulations in the high Knudsen number limit where the coefficient of restitution is not close to 1 , and the frequency of binary collisions is small compared to the frequency of particle-wall collisions. The objective of the present simulations is to determine the particle velocity distribution in the low and high Knudsen limits, and to compare them with theoretical predictions in both limits. A realistic wall boundary condition with normal and tangential coefficients of restitution is assumed, and the present boundary conditions differ from the bumpy wall conditions used in earlier studies in the low Knudsen number limit. Thus, these provide some indication of the effect of boundary conditions on the profiles in this limit. 


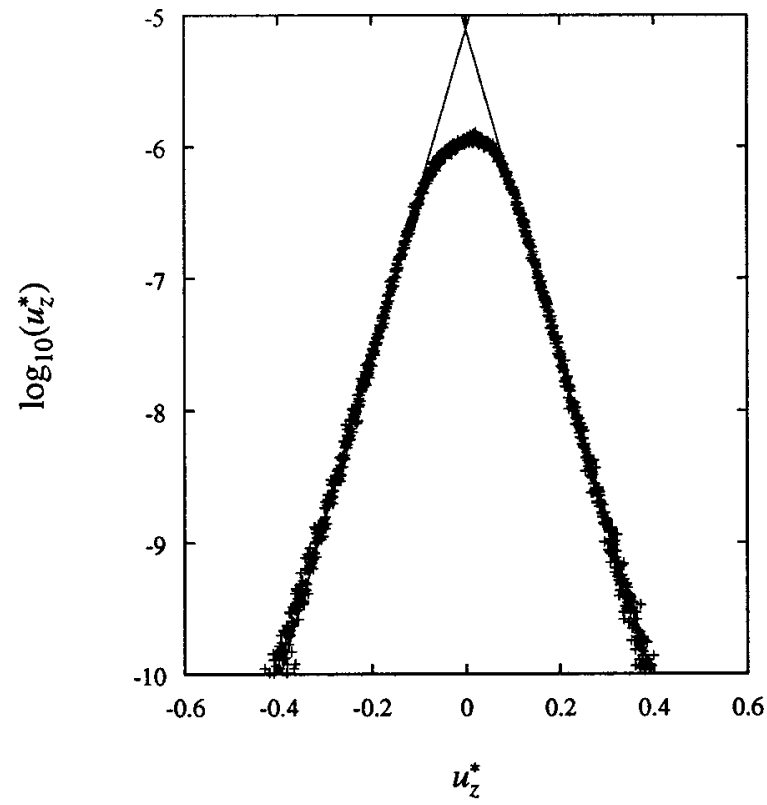

FIG. 8. Distribution of the cross-stream velocity for $e_{\mathrm{t}}=e_{\mathrm{n}}$ $=0.9, e=1.0$, and $\epsilon=0.01$ near the wall plotted on a log-linear scale.

Molecular dynamics methods are a valuable tool for simulating granular flows, and there have been many such studies. While Campbell and co-workers [12-14] used the hard sphere molecular dynamics, the soft particle contact model in which the overlap between particles is interpreted as a deformation that generates a restoring force proportional to both the amount of overlap and relative velocity of the particles at contact was adapted in Ref. [15]. These models study the behavior of idealized granular systems by calculating the motion of individual particles as they interact with each other and the walls. The effects of different kinds of boundaries were studied in detail [12,14,16,17], and the stress tensor and self-diffusivity in a sheared granular material have been determined $[13,18]$. There are several other studies in the literature (see Refs. [19-21] and references therein), focusing on the clustering, interaction between clusters and normal stress difference in sheared granular system.

The difficulty in an analytical determination of the distribution function for a wide range of parameters makes it im-

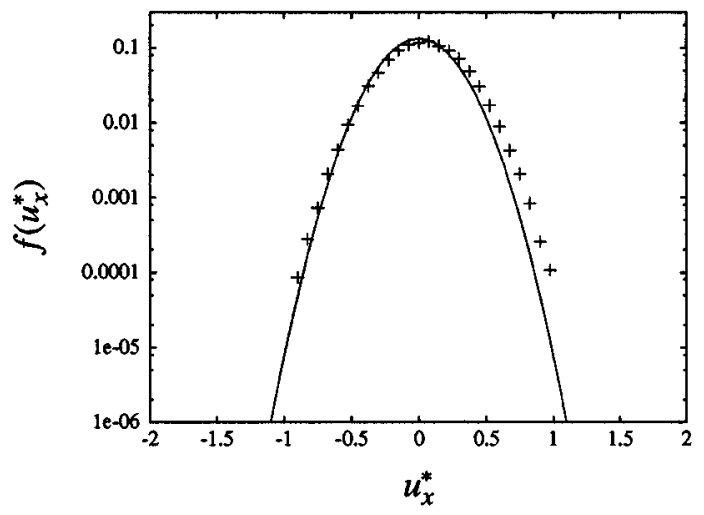

(a)

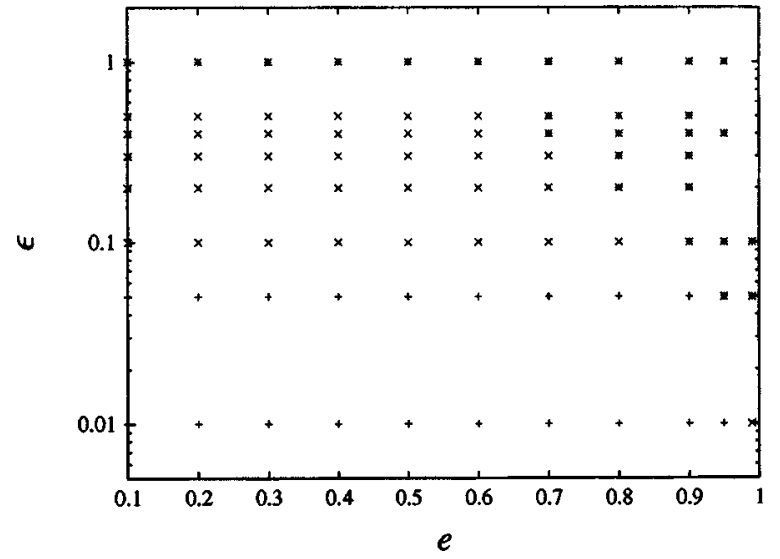

FIG. 9. Domains in $\epsilon-e$ parameter space for different types of distribution function. (+) - bimodal streamwise velocity distribution, $(\times)$ — streamwise velocity distribution with three maxima near the wall, and $(*)$-composite Gaussian streamwise velocity distribution.

portant to determine the detailed velocity distribution through the particle level simulation and experiment. There have been several studies available in literature that examine the distribution of particle velocities in different granular systems like shaken granular media and particles in a homogeneously cooling state (HCS) [22]. Non-Gaussian distribution functions of the vertical component of particle velocities subjected to vertical shaking have been reported [23,24], and the distribution of the horizontal component of particle velocities in a shaken circular cylinder have been observed [25]. The velocity in a heated granular medium has been found to have a non-Gaussian high velocity behavior [26]. A crossover from a non-Gaussian to a Gaussian behavior of the distribution of the horizontal component of particle velocities reported [27] as the degree of fluidization is varied in a horizontally vibrated granular media. In this work we present the distribution of both cross stream and streamwise components of the velocity of particles sheared in a two-dimensional channel.

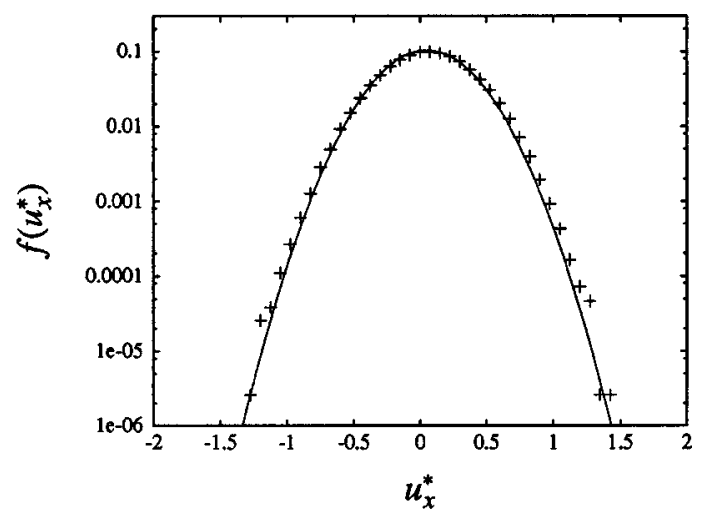

(b)

FIG. 10. Distribution function plotted in a log-linear scale for $e=0.99$, with the Gaussian fit using no adjustable fitting parameters for (a) $\epsilon=1.0$ and (b) $\epsilon=22.75$. (+) represents the simulation, and the (-) is the Gaussian fit. 


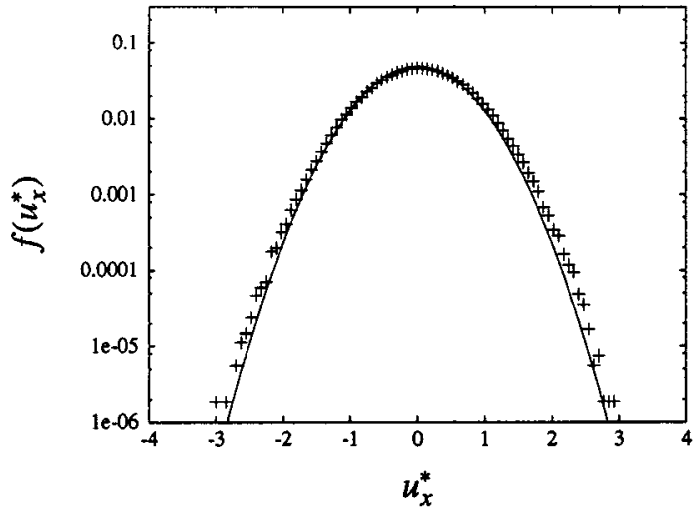

(a) $\epsilon=9.75$

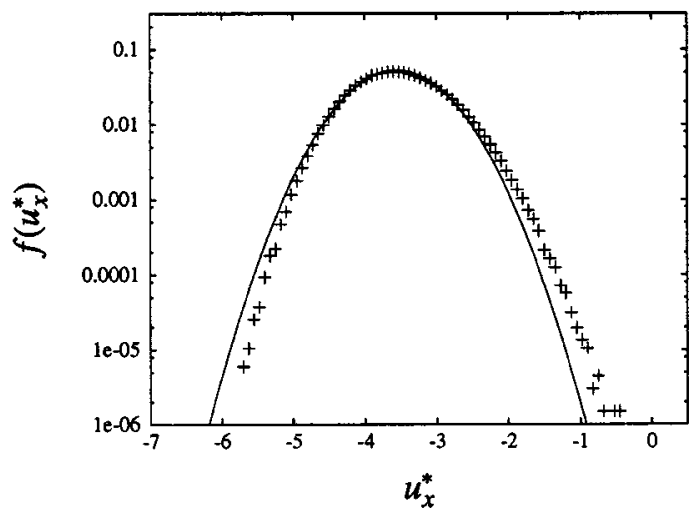

(c) $\epsilon=9.75$

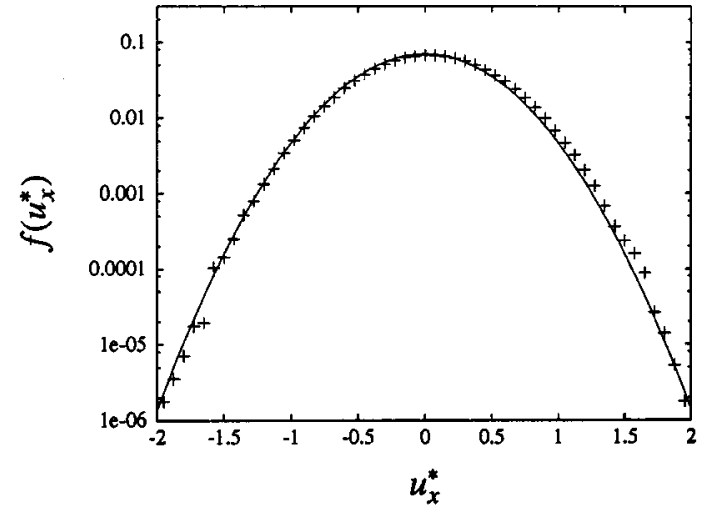

(b) $\epsilon=26.0$

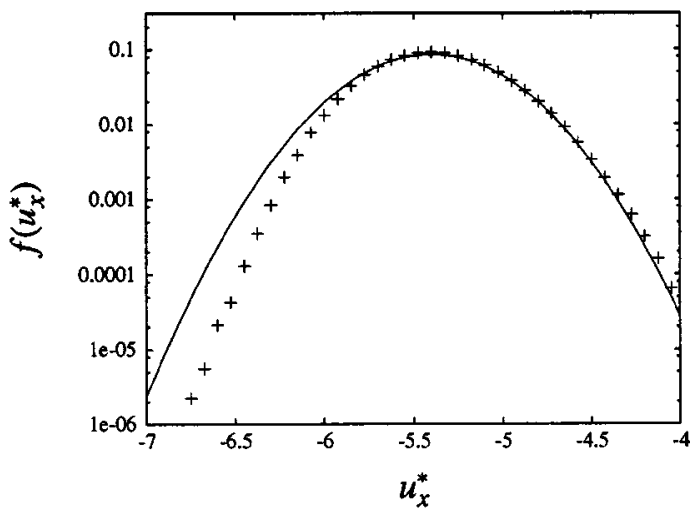

(d) $\epsilon=26.0$

FIG. 11. Distribution of the streamwise velocity at the centere and near the bottom wall of the channel for two different $\epsilon$. The coefficient of restitution is 0.9 for all the cases shown here. (a) and (b) at the centre of the channel for $\epsilon=9.75$ and 26.0, respectively, and (c) and (d) at $z / L=-0.95$ for $\epsilon=9.75$ and 26.0 , respectively.

\section{SIMULATION TECHNIQUE}

The system consists of a two-dimensional channel of width $L$ which contains disks of radius $\sigma$, and $n$ is the number density per unit width of the disks. The channel is bounded by moving walls with velocities $\pm V_{\mathrm{w}}$ at $z= \pm L$, as shown in Fig. 1, while periodic boundary conditions are used in the flow $(x)$ direction. The momentum imparted to the

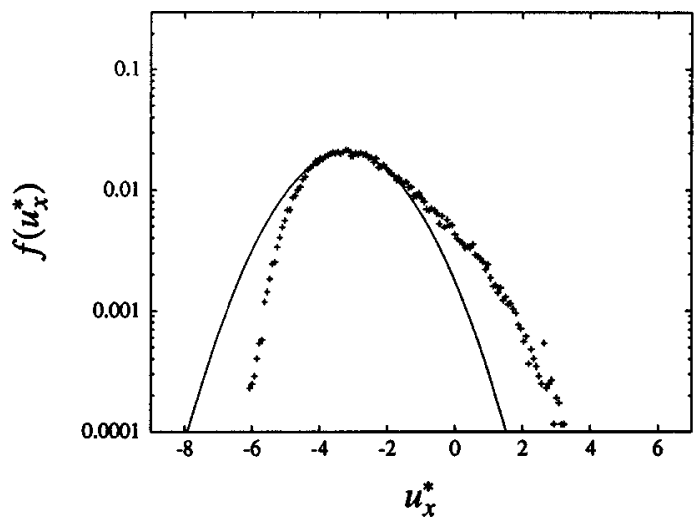

(a) $\epsilon=1.0$ particles by the walls results in a gradient in the mean velocity in the gradient $(z)$ direction. The change in the particle velocity due to a collision with the wall is given by

$$
u_{x}^{\prime}=e_{\mathrm{t}} u_{x} \pm\left(1-e_{\mathrm{t}}\right) V_{\mathrm{w}},
$$

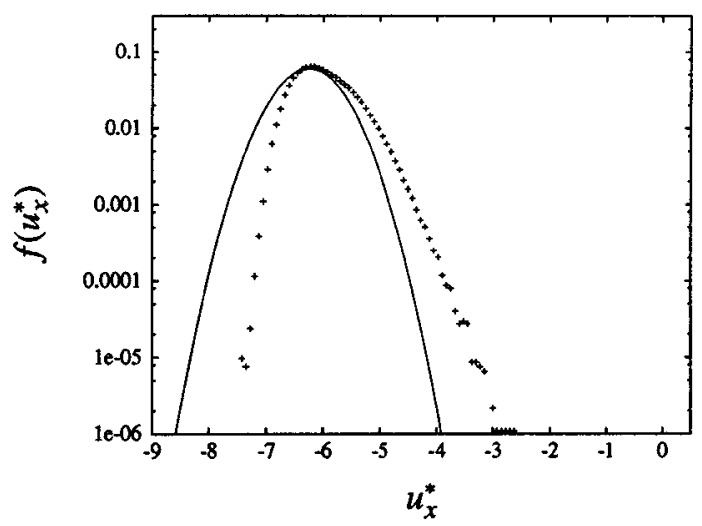

(b) $\epsilon=9.75$

FIG. 12. The distribution of the streamwise velocity near the bottom wall plotted on a log linear scale for $e=e_{\mathrm{t}}=e_{\mathrm{n}}=0.7$ and two different number densities of particles. 


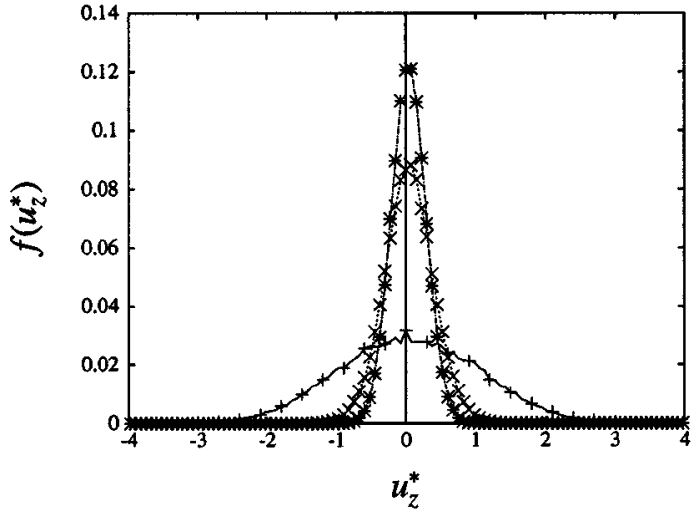

(a)

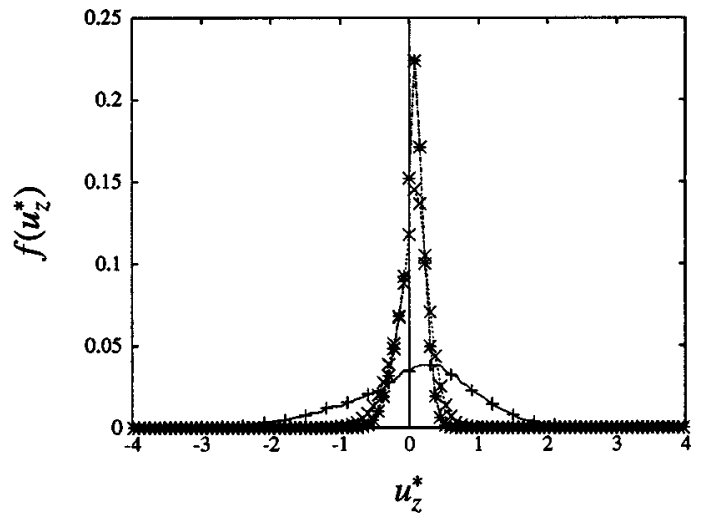

(b)

FIG. 13. Distribution of the cross-stream velocity at the center (a) and near the wall (b) of the channel for $e=0.7$. $(+) \epsilon=1.0,(\times) \epsilon$ $=13.0$, and $(*) \epsilon=26.0$.

$$
u_{z}^{\prime}=-e_{\mathrm{n}} u_{z}
$$

where $u_{x}^{\prime}$ and $u_{z}^{\prime}$ are the post collisional velocities of a particle with initial velocity $u_{x}$ and $u_{z}$, and $e_{\mathrm{t}}$ and $e_{\mathrm{n}}$ are tangential and normal coefficient of restitution respectively. The velocities of the particles before and after a binary collision are related as follows. The velocity of the center of mass, $\mathbf{v}=\mathbf{u}_{1}+\mathbf{u}_{2}$ remains unchanged, while the velocity difference $\mathbf{w}^{\prime}=\mathbf{u}_{1}^{\prime}-\mathbf{u}_{2}^{\prime}$ after the collision is related to the velocity $\mathbf{w}$ $=\mathbf{u}_{1}-\mathbf{u}_{2}$ before the collision by

$$
\mathbf{w}^{\prime}=[\mathrm{I}-(1+e) \mathbf{k} \mathbf{k}] \cdot \mathbf{w},
$$

where $e$ the coefficient of restitution for binary collisions, and $\mathbf{k}$ is directed along the line joining the centers of two colliding particles.

The event driven simulation consists of a streaming step and a collision step. In the streaming step, the time required for the next collision is calculated based on the current particle positions and velocities, and the positions are advanced along a straight line up to the next collision. In the collision step, the velocity of the colliding particles is updated according to Eq. (3). Unlike in studies on infinite systems, it is necessary to consider the possibility of particle-wall colli-

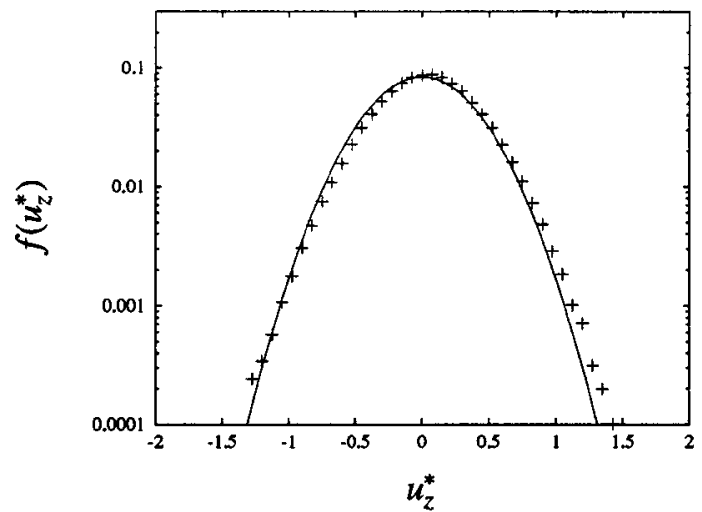

(a) sions as well in the present case while calculating the time required for the next collision. The collision rules for the particle-wall collisions are given by Eqs. (1) and (2), while the collision rules for particle-particle collisions are the standard ones where the velocity of the center of mass is unchanged, while the relative velocity after collision is related to that before collision by Eq. (3).

\section{HIGH KNUDSEN NUMBER LIMIT}

The velocity distribution in the high Knudsen number limit for the flow in a channel with boundary conditions (1) and (2) for particle-wall collisions was calculated by Kumaran [11] in the limit where the frequency of particle-particle collisions is small compared to the frequency of wall-particle collisions. The frequency of wall-particle collisions per unit width is proportional to $n u$, where $n$ is the number of particles per unit area and $u$ is the magnitude of the particle velocity, whereas the frequency of wall-particle collisions per unit width of the channel is proportional to $n^{2} \sigma u L$, since $n^{2} u \sigma$ is the frequency of binary collisions per unit area. Therefore, the ratio of the frequencies of particle-particle and wall-particle collisions is given by the dimensionless param-

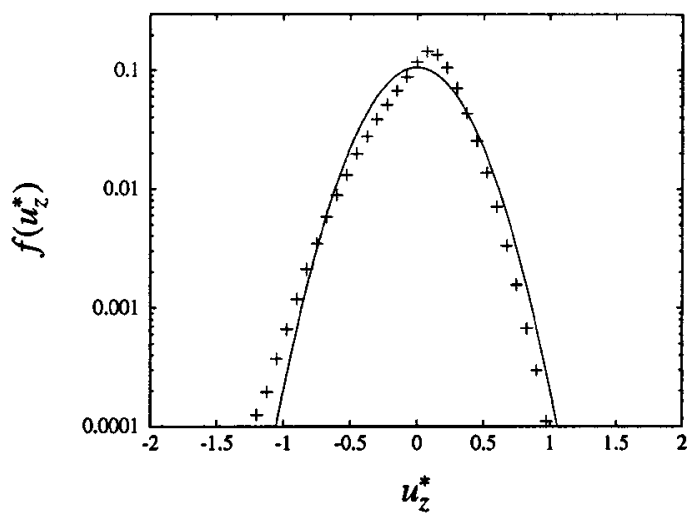

(b)

FIG. 14. Distribution of $u_{z}$ on log-linear scale at the center (a) and near the wall (b) of the channel for a system with $e=0.7$ and $\epsilon$ =13.0. The solid line is a Gaussian fit with no adjustable parameter and the points are obtained from simulation. 


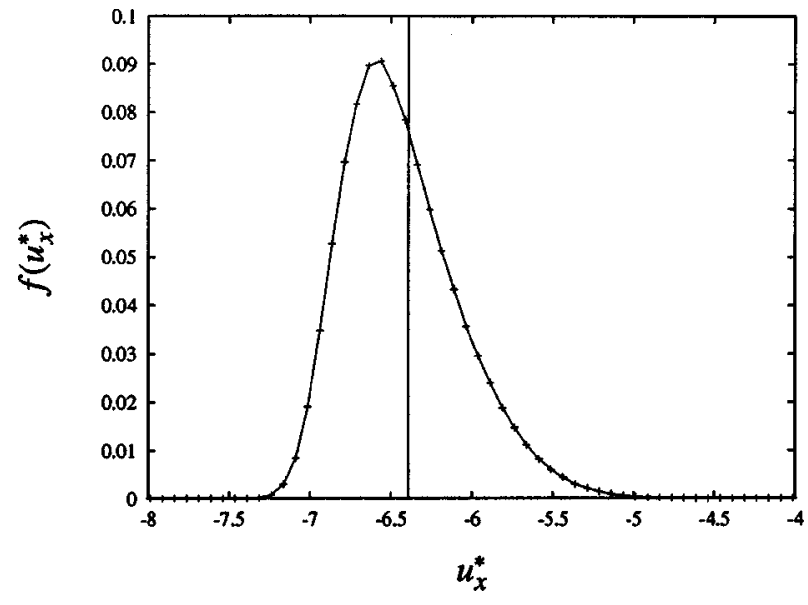

FIG. 15. Distribution of the streamwise velocity for $e=0.7$ and $\epsilon=13.0$, i.e., $\mathrm{Kn}=0.077$, at the center of the channel. The curve is fit by a "composite Gaussian" with two Gaussian distributions with different variances patched at their maxima. The straight line shows the mean value.

eter $\epsilon \equiv n \sigma L$, which is small compared to 1 in this limit. An asymptotic analysis is used where binary collisions are neglected in the leading approximation, and the situation corresponds to a single particle in a channel with boundary conditions (1) and (2). If a particle has velocity $\left(u_{x}, u_{z}\right)$ and collides with the top wall, the post-collisional velocities are

$$
\begin{gathered}
u_{x}^{\prime}=e_{t} u_{x}+\left(1-e_{t}\right) V_{w} \\
u_{z}^{\prime}=-e_{n} u_{z} .
\end{gathered}
$$

This particle now has a negative $u_{z}$, and subsequently collides with the bottom wall. The velocities after the second collisions are

$$
u_{x}^{\prime \prime}=e_{t} u_{x}^{\prime}-\left(1-e_{t}\right) V_{w}=e_{t}^{2} u_{x}-\left(1-e_{t}\right)^{2} V_{w},
$$

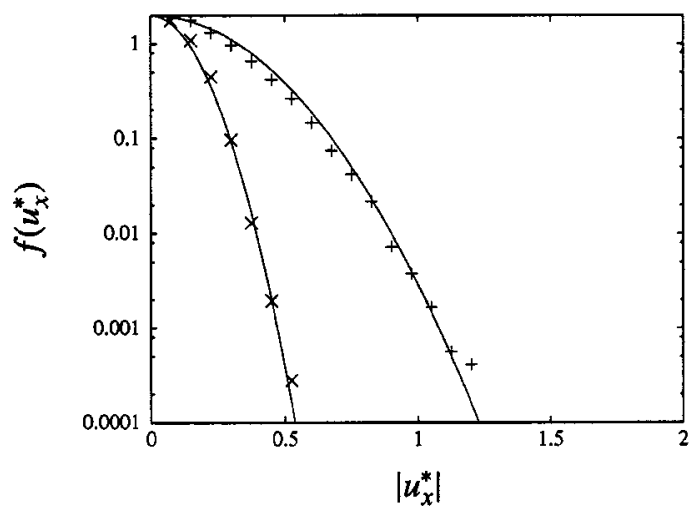

(a)

$$
u_{z}^{\prime \prime}=e_{n}^{2} u_{z} .
$$

A steady state is achieved if the particle returns to its original value after two collisions, or $u_{x}^{\prime \prime}=u_{x}$ and $u_{z}^{\prime \prime}=u_{z}$. It is easy to see, from Eqs. (4) and (5), that the particle velocity returns to its original value after two collisions only if the velocities are

$$
\begin{gathered}
u_{x}=-V=\frac{-\left(1-e_{t}\right) V_{w}}{1+e_{t}}, \\
u_{x}^{\prime}=V=\frac{\left(1-e_{t}\right) V_{w}}{1+e_{t}}, \\
u_{z}=u_{z}^{\prime}=0 .
\end{gathered}
$$

Further, an analytical expression can be obtained for the velocity $\left(u_{x}^{(n)}, u_{z}^{(n)}\right)$ after $n$ successive collisions with the walls if the initial velocity is $\left(u_{x}^{(0)}, u_{z}^{(0)}\right)$ :

$$
\begin{gathered}
u_{x}^{(n)}+(-1)^{n}\left(1+(-1)^{(n-1)} e_{t}^{n}\right) V=e_{t}^{n} u_{x}^{(0)} \\
u_{z}^{(n)}=(-1)^{n} e_{n}^{n} u_{z}^{(0)} .
\end{gathered}
$$

Thus, if the particle velocities are initially random, with equal numbers of particles having positive and negative $z$ velocities, then the particle velocities after a large number of collisions converge to the velocities $( \pm V, 0)$. In this case, the distribution function for the particle velocities consists of two delta functions at $( \pm V, 0)$.

However, as the particle velocity in the $z$ direction reduces to zero, the frequency of the particle-wall collisions, which is proportional to $u_{z}$, also reduces, and it is necessary to include the effect of binary collisions. This is incorporated by assuming that binary collisions occur between particles with velocity $( \pm V, 0)$. The reason for only considering binary collisions between particles with velocities $( \pm V, 0)$. is the following. For particles whose velocity $u_{z}$ is $O(V)$, there is a significant error in the calculation of the post-collisional velocities if we assume that the particle velocities are $( \pm V, 0)$. However, for these particles, the frequency of binary colli-

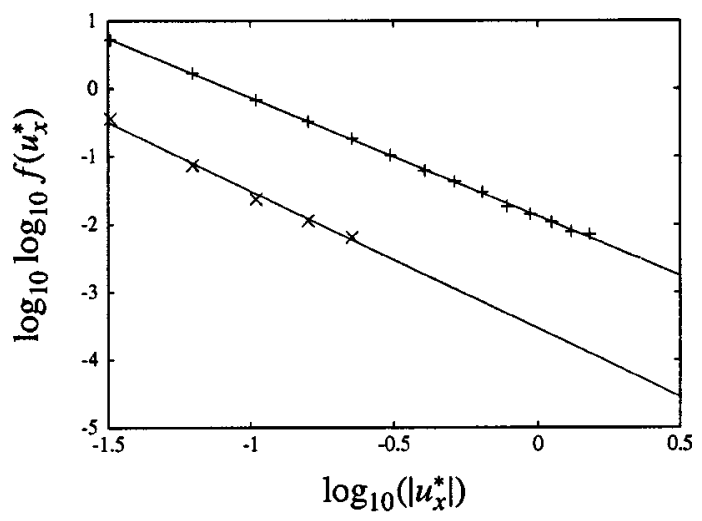

(b)

FIG. 16. Distribution function for streamwise velocity for $e=0.7$ and $\epsilon=26.0$ at the wall of the channel plotted on a log-linear scale (a). The two distinct lines indicate two variances below and above the maximum. (b) shows the fit of the distribution function to Eq. (10) in the $\log$ scale. 


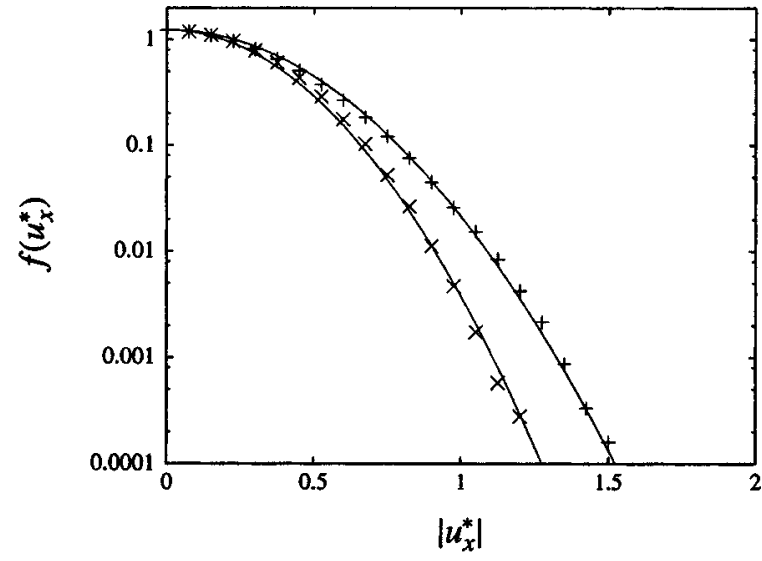

(a) $\epsilon=26.0$

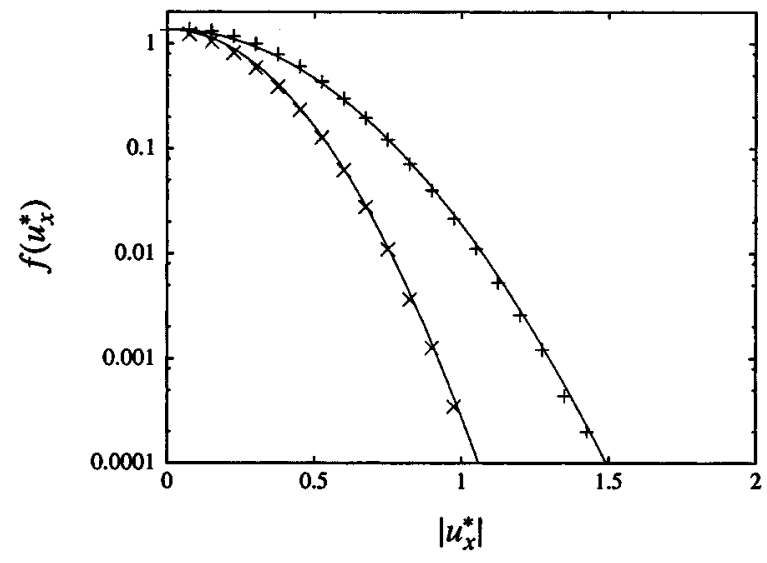

(b) $\epsilon=30.0$

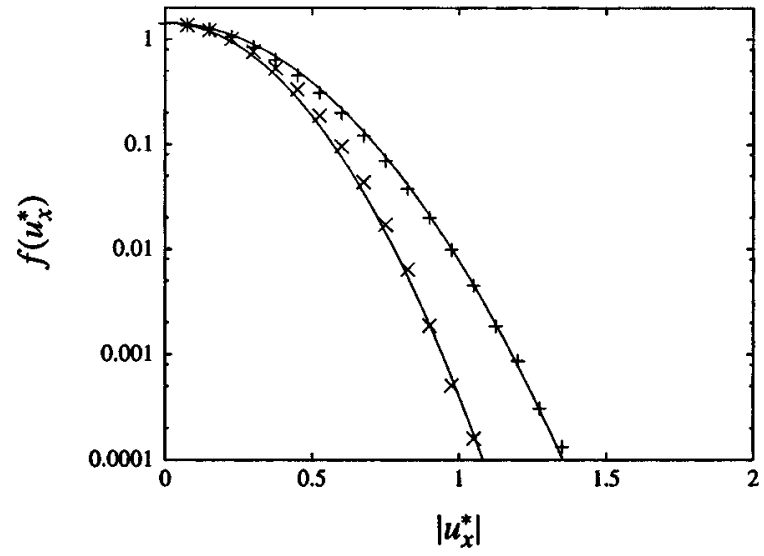

(c) $\epsilon=34.0$

FIG. 17. Distribution of the streamwise velocity on a log-linear scale with a corresponding Gaussian fit with no adjustable parameters for $e=0.9$ near wall. The two distinct segments indicate the presence of two variances below and above maxima. (+) represents the variance above the maxima and $(X)$ represents the variance below the maxima.

sions is $O(\epsilon)$ smaller than that of particle-wall collisions, and so the error incurred in the calculation of the distribution function is $O(\epsilon)$. For particles whose velocities are $O(\epsilon V)$, different from $( \pm V, 0)$, the frequency of binary collisions is of the same magnitude as that of particle-wall collisions, but there is only a small error of $O(\epsilon)$ in the calculation of the distribution function due to the assumption that the particles have velocities $( \pm V, 0)$. Thus, this approximation regarding the velocities of the particles before a binary collision incurs only a small error throughout the domain.

An elastic binary collision between two particles at $( \pm V, 0)$ scatters these particles onto a circle in velocity space with a center at the origin and a radius $V$. These particles undergo collisions with the wall, since they have a nonzero velocity in the $z$ directions. These particle-wall collisions transport the particles onto a series of discrete contours in velocity space, until their velocity returns to a region of size $O(\epsilon V)$ about $( \pm V, 0)$. The flux of particles entering and leaving a differential segment along a contour is determined using the Boltzmann collision operator for binary collisions, and the distribution function along these contours at steady state is determined by balancing the flux of particles entering the contour with the flux leaving. The normal and shear stresses are calculated from the distribution function. Details are provided in the earlier paper [11].

It is easy to see that, in the leading approximation, which corresponds to two delta functions at $( \pm V, 0)$, the second moment of the velocity distribution (which is proportional to the stress) is highly anisotropic:

$$
\begin{gathered}
\left\langle u_{x}^{2}\right\rangle=V^{2}, \\
\left\langle u_{z}^{2}\right\rangle=0, \\
\left\langle u_{x} u_{z}\right\rangle=0 .
\end{gathered}
$$

However, when the velocities of particles scattered due to binary collisions are included, the second moments of the velocity distribution are found to scale as follows:

$$
\begin{gathered}
\left\langle u_{x}^{2}\right\rangle-V^{2} \sim \epsilon, \\
\left\langle u_{z}^{2}\right\rangle \sim \epsilon, \\
\left\langle u_{x} u_{z}\right\rangle \sim \epsilon \log (\epsilon) .
\end{gathered}
$$

It should be noted that the logarithmic dependence of $\left\langle u_{x} u_{z}\right\rangle$ is difficult to discern in simulations, since it is necessary to go to very small values of $\epsilon$ to recover this, but the dependence on $\epsilon$ in the high Knudsen number limit can be verified.

The simulation has been carried out for three sets of $e_{\mathrm{n}}$ and $e_{\mathrm{t}}$, while the coefficient of restitution for particle particle collisions $e$ has been set equal to 1 . The simulation can be easily extended to cases where $e \neq 1$. Figures 2 and 3 show the distribution functions for different sets of values of the coefficient of restitution in normal and tangential direction, as a function of $\epsilon$ at two different points across the channel width. The distribution function is very different from Maxwell-Boltzmann distribution, but converges to the predictions of the asymptotic analysis [11] in the limit $\epsilon \rightarrow 0$.

It is observed that the distribution function is bimodal (Figs. 2 and 3), and that the peaks occur at $\pm V$ as predicted by the asymptotic analysis, where $V=V_{\mathrm{w}}\left(1-e_{\mathrm{t}}\right) /\left(1+e_{\mathrm{t}}\right)$ and $V_{\mathrm{w}}$ is the wall velocity. It is also seen from the figure that the 


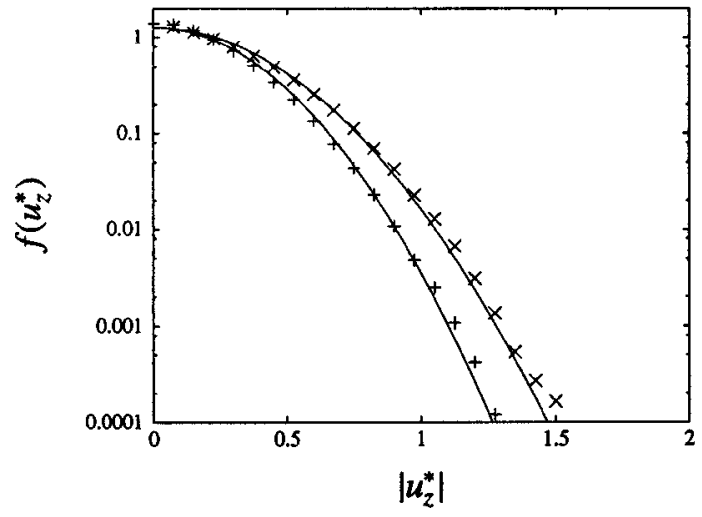

(a) $\epsilon=26.0$

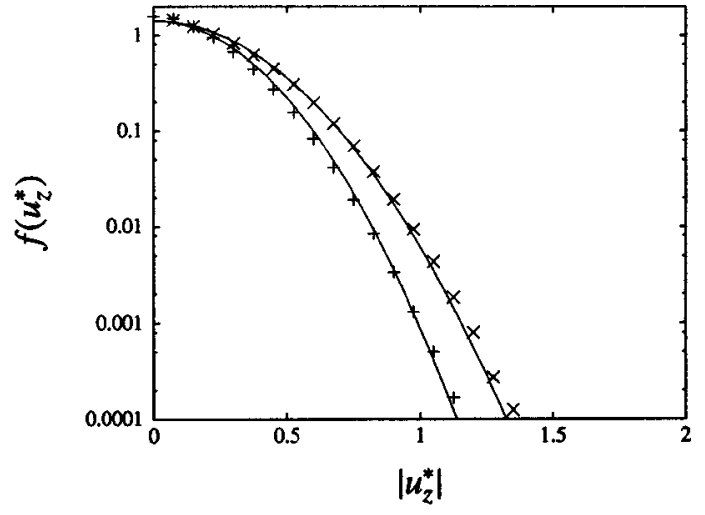

(b) $\epsilon=30.0$

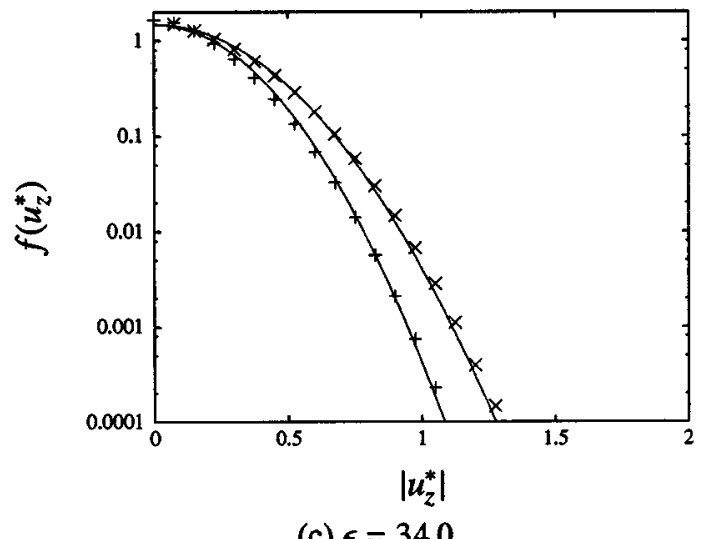

FIG. 18. Distribution of the cross-stream velocity on a log-linear scale with a corresponding Gaussian fit with no adjustable parameters for $e=0.9$ near the wall. The two distinct segments indicate the presence of two variances below and above the maxima. ( +$)$ represents the variance above the maxima and $(X)$ represents the variance below the maxima.

height of the peak decreases and the width increases as $\epsilon$ increases. However, the bimodal nature of the distribution function persists for $\epsilon$ up to 0.05 .

Different moments of scaled velocity $\psi\left(\mathbf{u}^{*}\right)$ were determined in the simulation, where $\mathbf{u}^{*}=(\mathbf{u} / V)$ is the velocity scaled by $V$. Figure 4 shows the scaling of $\left\langle u_{z}^{* 2}\right\rangle$ and $\left\langle u_{x}^{* 2}\right\rangle$ -1 as a function of $\epsilon$. The theory predicts that $\lim _{\epsilon \rightarrow 0}\left(\left\langle u_{x}^{* 2}\right\rangle\right.$ $-1) \propto \epsilon$ and $\lim _{\epsilon \rightarrow 0}\left\langle u_{z}^{* 2}\right\rangle \propto \epsilon$; these scaling laws are in agreement with the simulation results. The moments of the distribution function are shown as a function of position in Fig. 5 . It is observed that the difference between the wall and particle velocities at the wall increases as $e_{\mathrm{t}}$ increases, as can be

TABLE I. Temperature obtained from simulation compared with that obtained using the theory of Ref. [3].

\begin{tabular}{lccccc}
\hline \hline \multicolumn{1}{c}{$v$} & $e$ & $\begin{array}{c}\sigma \\
(\mathrm{m})\end{array}$ & $\begin{array}{c}V_{\mathrm{w}} / L \\
(1 / \mathrm{s})\end{array}$ & $\begin{array}{c}T_{\text {Rich }} \\
\left(\mathrm{m}^{2} / \mathrm{s}^{2}\right)\end{array}$ & $\begin{array}{c}T_{\text {sim }} \\
\left(\mathrm{m}^{2} / \mathrm{s}^{2}\right)\end{array}$ \\
\hline 0.103 & 0.99 & 0.02 & 0.7 & 0.0848 & 0.0856 \\
0.46 & 0.99 & 0.045 & 1.25 & 0.089 & 0.088 \\
0.0975 & 0.9 & 0.02 & 4.0 & 0.292 & 0.291 \\
0.4355 & 0.9 & 0.045 & 5.67 & 0.181 & 0.183 \\
0.094 & 0.7 & 0.02 & 6.0 & 0.2217 & 0.218 \\
0.445 & 0.7 & 0.045 & 7.0 & 0.0769 & 0.08 \\
\hline \hline
\end{tabular}

inferred from the relation between the wall velocity and post collisional particle velocity, and this difference decreases as $\epsilon$ increases, because a particle undergoes multiple binary collisions before reaching the wall, and the mean velocity equilibrates to the wall velocity. It is also observed in Fig. 5 that the second moments $\left(\left\langle u_{x}^{2}\right\rangle\right.$ and $\left.\left\langle u_{z}^{2}\right\rangle\right)$ are anisotropic in this limit and that the anisotropy decreases with an increase in $\epsilon$.

\section{LOW KNUDSEN NUMBER LIMIT}

It has been observed that the bimodal nature of the distribution function for the streamwise velocity changes as $\epsilon$ is increased, or $\mathrm{Kn}$ is decreased. In this section, the particle velocity distribution in the low Kn limit will be discussed. For all the results presented here, the tangential and normal coefficient of restitution for wall particle collisions are assumed to be equal to the restitution coefficient for the binary collisions. Figure 6 shows the distribution function for both the streamwise and cross-stream velocities of particles for different values of $\epsilon$ for a fixed coefficient of restitution at two different points in the channel, and Fig. 7 shows the velocity distribution functions for a fixed $\epsilon$ but a different coefficient of restitution at two different points in the channel. It is observed that the distribution function for the streamwise velocity changes from a bimodal to a unimodal 


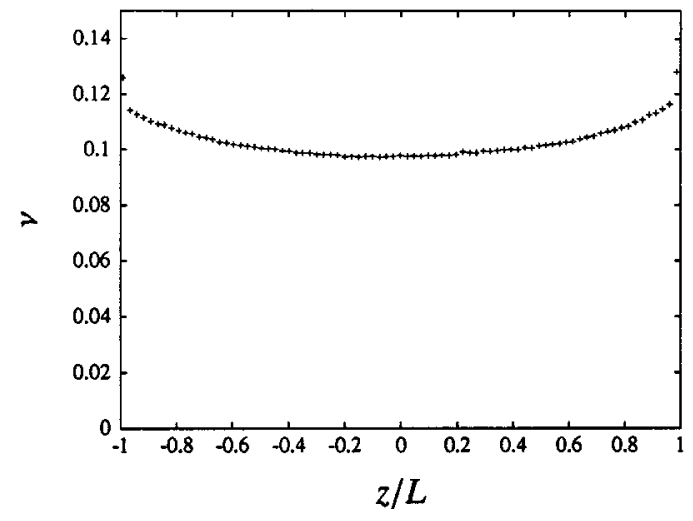

(a)

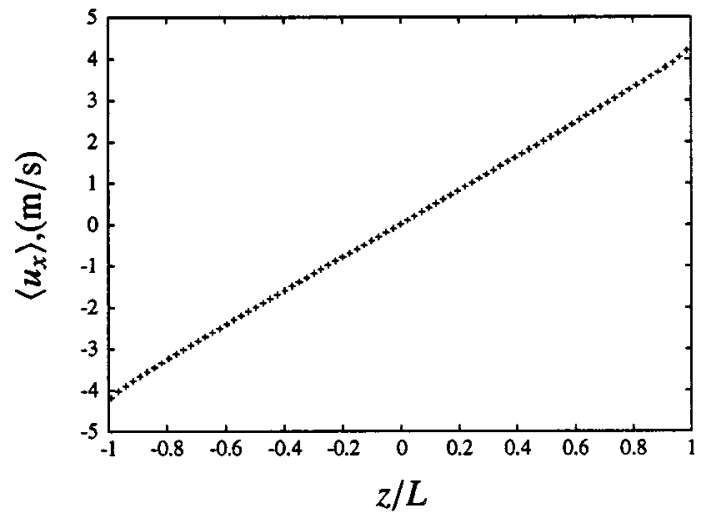

(b)

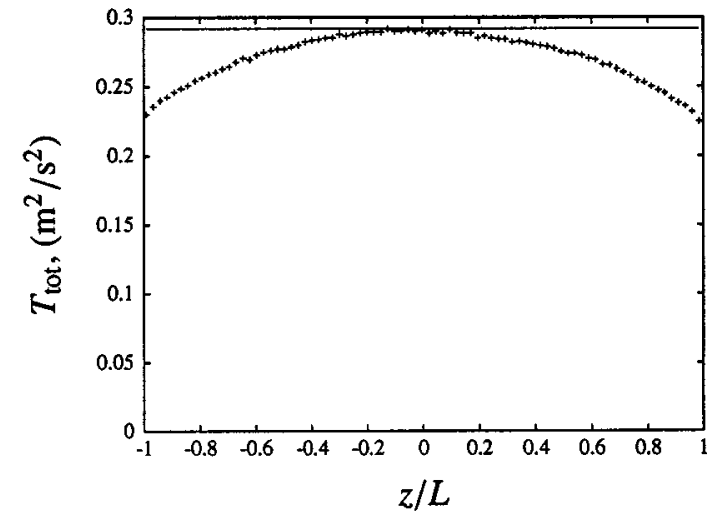

(c)

FIG. 19. (a) Density, (b) mean velocity, and (c) temperature for $e=0.9$ and $\epsilon=13.0$. (-) represents the temperature obtained in Ref. [3] for the given density and the strain rate.

distribution as $\epsilon$ is increased. It is also observed that in the high Knudsen number limit, the distribution of the streamwise velocity of particles is bimodal for any restitution coefficient and at any point across the channel. However this bimodal nature disappears as the $\epsilon$ approaches 1 . There exists a transition zone where the distribution of streamwise velocity is bimodal at the center of the channel, and has three maxima near the wall (Fig. 7). The third peak in the distribution function appears at a velocity scaled $\left[\left(1-e_{\mathrm{t}}\right) /\right.$ $\left.\left.\left(1+e_{\mathrm{t}}\right)\right] V_{\mathrm{w}}\right\}$ beyond \pm 1 near the top and bottom walls of the channel. In this regime, the nature of the streamwise velocity distribution depends on the coefficient of restitution, as seen in Fig. 7. For $\epsilon \geqslant 1$ the velocity distribution is unimodal, but for $\epsilon<1$ the velocity distribution could be unimodal or bimodal depending on the coefficients of restitution. Further, the nature of the distribution also depends on the position in the channel. As the coefficient of restitution decreases, the distribution of $u_{x}$ deviates from the Gaussian distribution near the wall and exhibits skewness. At the center of the channel, however, the distribution function continues to be Gaussian.

Next, the cross-stream velocity distribution for the particle is studied. The distribution of the cross-stream velocity is a delta function in the high Knudsen limit and is unimodal in the moderate to low Knudsen number regime. The crossstream velocity distribution is non-Gaussian for systems with moderately high Knudsen number, and varies as $k \exp \left(-u_{z}\right)$ (Fig. 8).
In the limit where the Knudsen number is small compared to 1 , the distribution is Gaussian for a system with a restitution coefficient close to 1 . The distribution of the crossstream velocity of particles remains Gaussian at the center of the channel for a small coefficient of restitution, but assumes the form of a composite Gaussian near the wall, with different variances above and below the maximum velocity. The details of the scaling of the distribution functions and the anisotropy are discussed a little later. The regions in the $\epsilon$ $-e$ parameter space where the distributions for the streainwise velocity are bimodal, composte Gaussian, and transition type with more than one maximum are shown in Fig. 9.

In the low Knudsen number limit $(\epsilon>1)$ for $(1-e) \ll 1$, the distribution function is Gaussian, as shown in Fig. 10. In this limit the velocities in the streamwise direction have been nondimensionalized with the wall velocities $V_{w}$. There is no adjustable parameter used in the fit, and there is good agreement for five decades. There is a significant departure from the Gaussian distribution when the coefficient of restitution is not close to 1 . Figure 11 shows the distribution function for both the streamwise and cross-stream components of the velocity at two different positions in the gradient direction at $e=0.9$ in the low Knudsen number regime. It is observed that the distribution is symmetric at the center of the channel whereas there is skewness in the distribution function near the wall. It is apparent from Fig. 11 that the skewness is stronger as the Knudsen number is increased, or as $\epsilon$ is decreased, and from Fig. 12 that the skewness is stronger when 


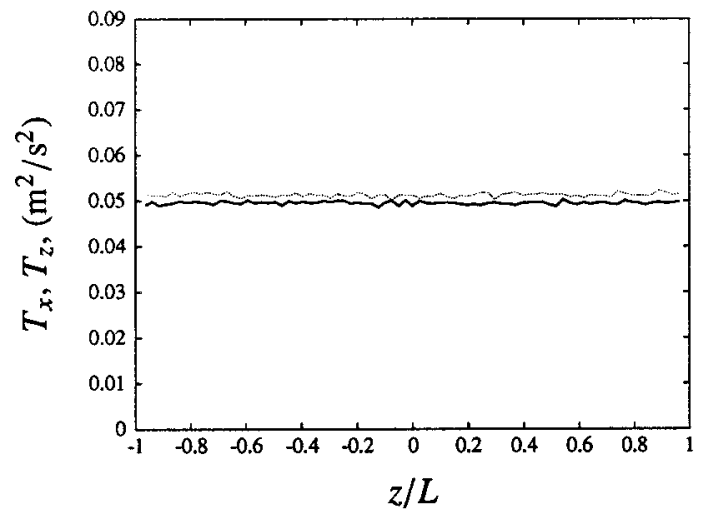

(a) $\epsilon=1.0, e=0.99$

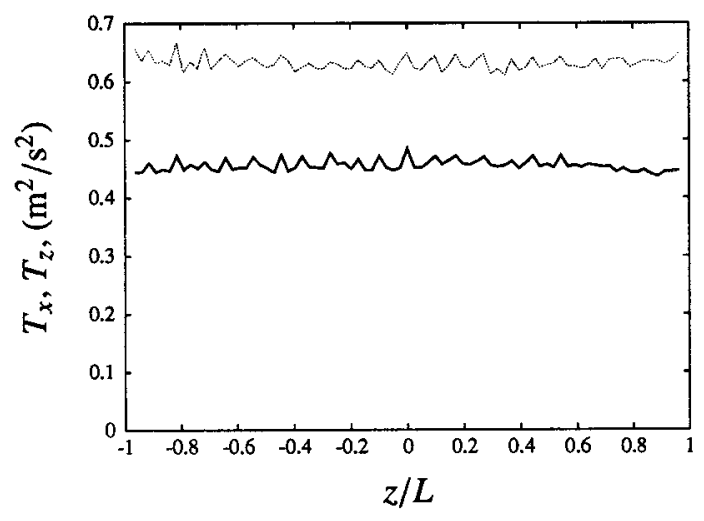

(c) $\epsilon=1.0, e=0.9$

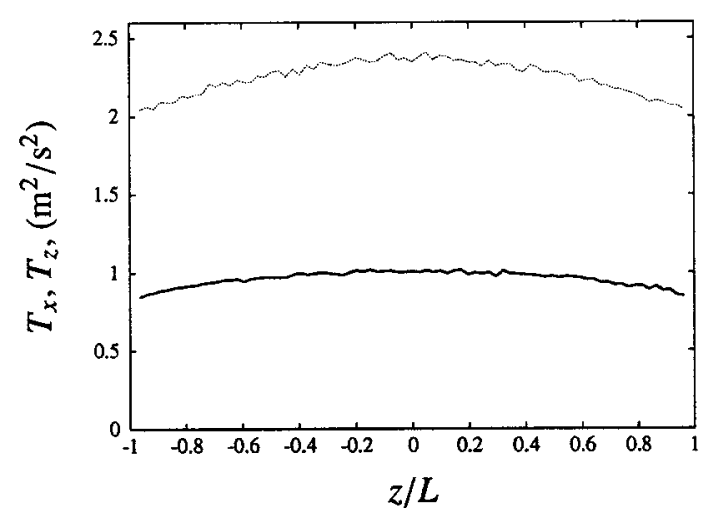

(e) $\epsilon=1.0, e=0.7$

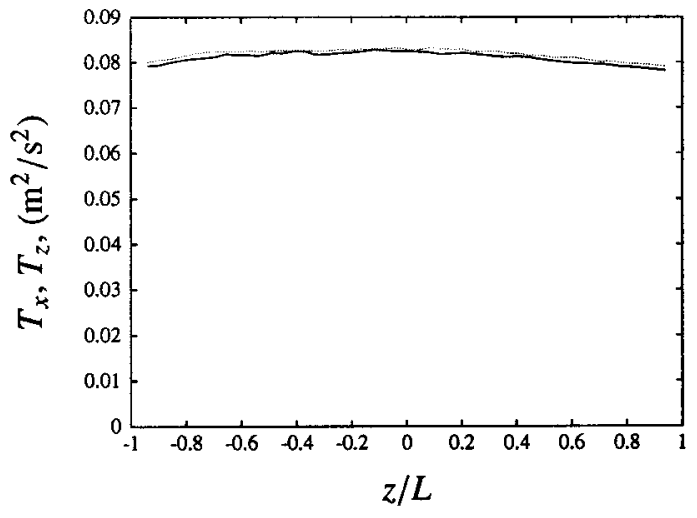

(b) $\epsilon=22.75, e=0.99$

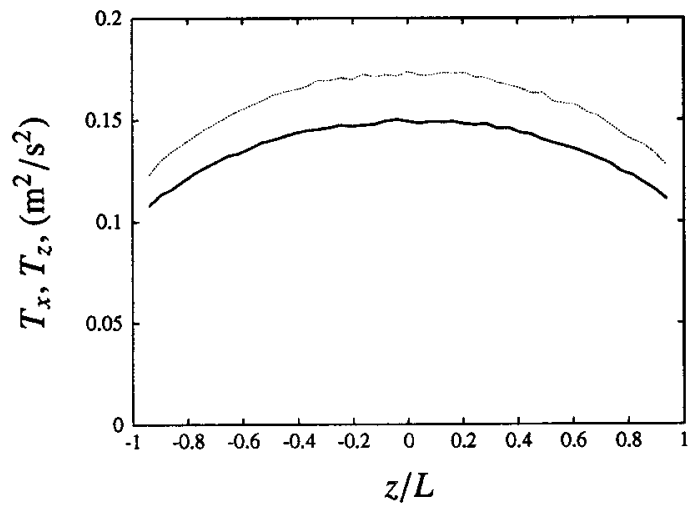

(d) $\epsilon=22.75, e=0.9$

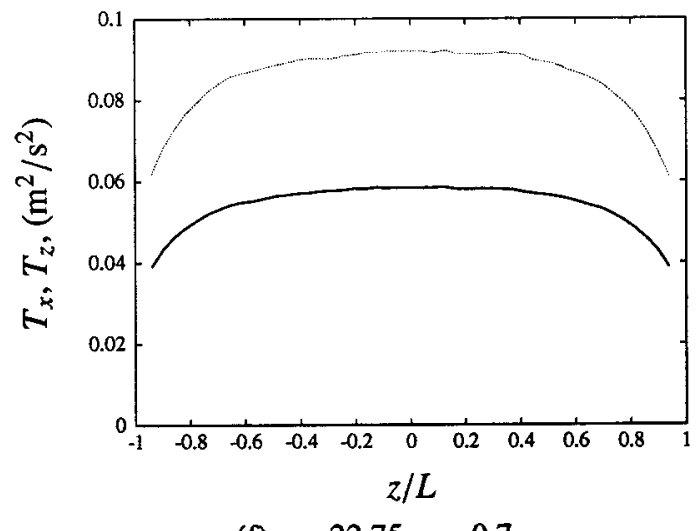

(f) $\epsilon=22.75, e=0.7$

FIG. 20. Anisotropy in temperature for different coefficients of restitution in the low Knudsen number regime, $(\ldots \ldots) T_{x}$ and $(-) T_{z}$.

$e$ is decreased at constant $\epsilon$. A similar trend is observed for the cross-stream component of velocity (Fig. 13).

The results obtained from the simulations in the low Knudsen number high inelasticity limit are further studied to obtain a quantitative description of the scaling of the distribution function at high velocities (Fig. 14). Fig.15 shows the distribution of the streamwise velocity of particles near the bottom wall of the channel. The distribution has a form

$$
f\left(u_{x}\right)=k_{1} \exp \frac{-\left(u_{x}-u_{x m}\right)^{n}}{k_{2}},
$$

where $u_{x m}$ is the position of the maximum of the distribution function, and $k_{1}$ and $n$ is determined from the intercept of the
TABLE II. Source and dissipation near wall as determined from the simulations.

\begin{tabular}{cccccccc}
\hline \hline $\begin{array}{c}\sigma \\
(\mathrm{m})\end{array}$ & $e$ & $\epsilon$ & $\begin{array}{c}T \\
\left(\mathrm{~m}^{2} / \mathrm{s}^{2}\right)\end{array}$ & $v$ & $\begin{array}{c}V_{w} / L \\
(1 / \mathrm{s})\end{array}$ & $\begin{array}{c}S o \\
\left(1 / \mathrm{s}^{3}\right)\end{array}$ & $\begin{array}{c}D o \\
\left(1 / \mathrm{s}^{3}\right)\end{array}$ \\
\hline 0.01 & 0.99 & 1.0 & 0.06 & 0.004 & 0.143 & 0.14 & 0.0134 \\
0.01 & 0.9 & 1.0 & 0.645 & 0.004 & 1.0 & 21.09 & 4.52 \\
0.01 & 0.7 & 1.0 & 1.68 & 0.0045 & 5.0 & 745.42 & 66.06 \\
0.035 & 0.99 & 22.75 & 0.0861 & 0.324 & 2.25 & 22.18 & 3.53 \\
0.035 & 0.9 & 22.75 & 0.125 & 0.36 & 5.75 & 176.74 & 72.93 \\
0.035 & 0.7 & 22.75 & 0.054 & 0.375 & 7.5 & 161.59 & 60.313 \\
\hline \hline
\end{tabular}




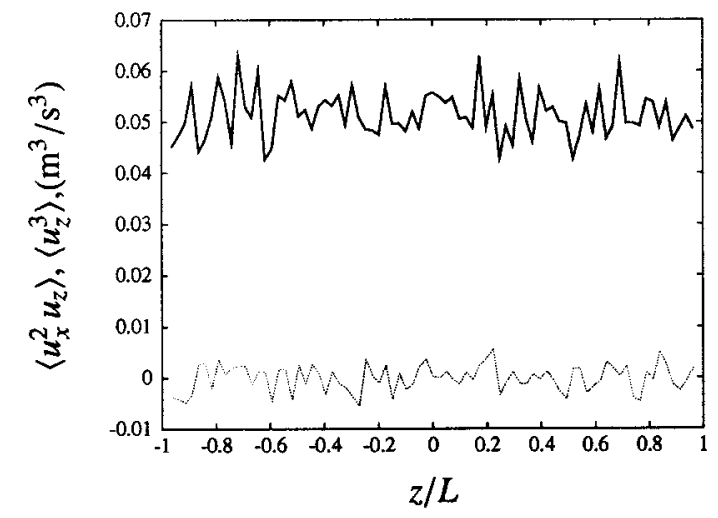

(a) $\epsilon=1.0, e=0.99$

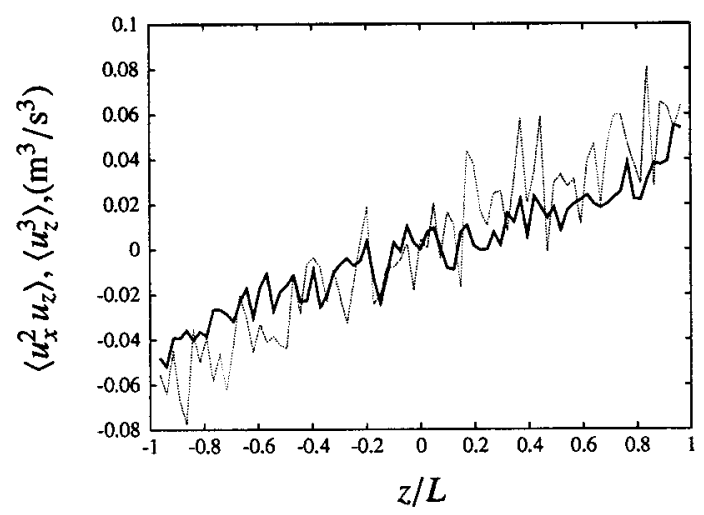

(c) $\epsilon=1.0, e=0.9$

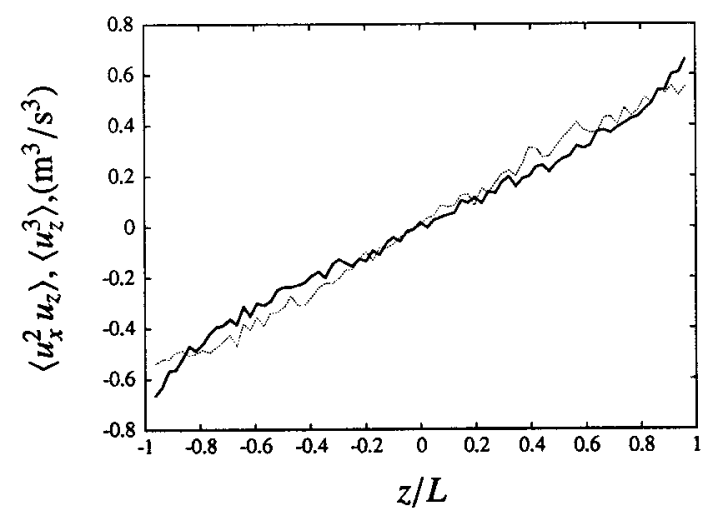

(e) $\epsilon=1.0, e=0.7$

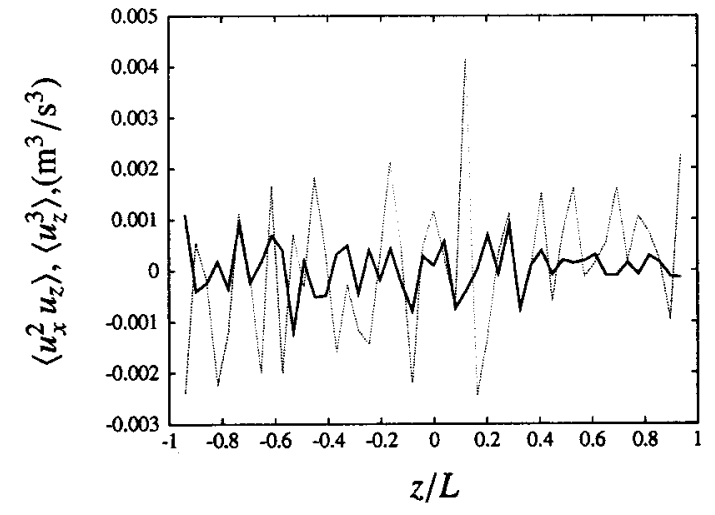

(b) $\epsilon=22.75, e=0.99$

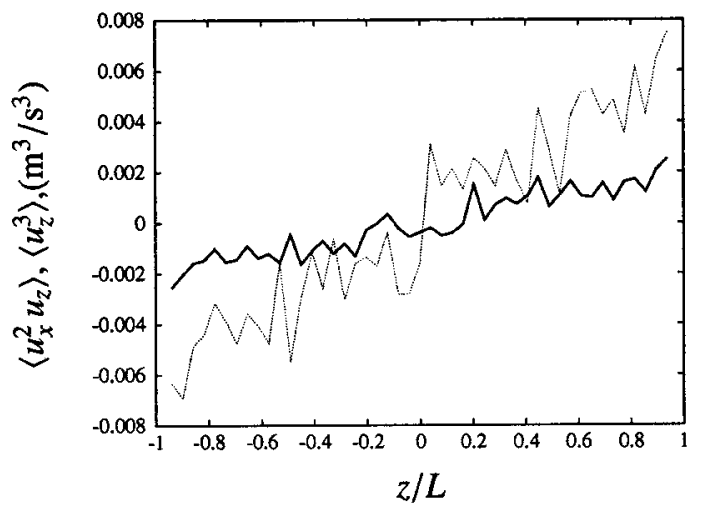

(d) $\epsilon=22.75, e=0.9$

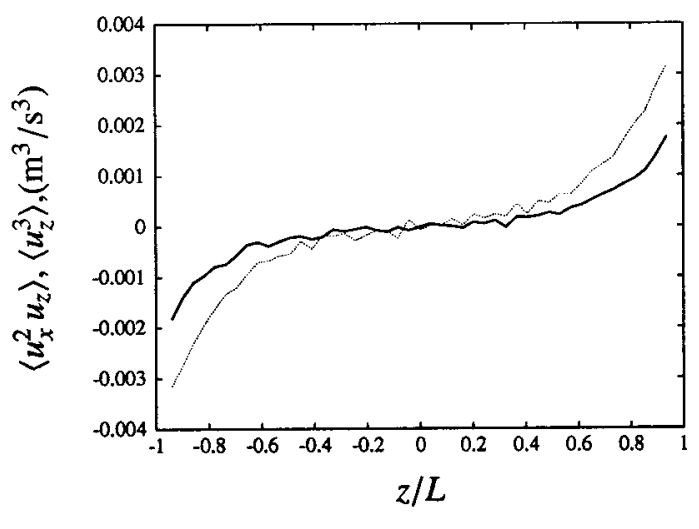

(f) $\epsilon=22.75, e=0.7$

FIG. 21. Flux of energy for different coefficients of restitution in the low Knudsen number regime $(\ldots \ldots)\left\langle u_{x}^{2} u_{z}\right\rangle,(-)\left\langle u_{z}^{3}\right\rangle$.

function plotted in a log linear scale. It should be noted that Eq. (10) is just a fit to the observed form of the distribution function, which incorporates the possibility of different velocity variances in the limits $u_{x}=+\infty$ and $u_{x}=-\infty$. It is assumed here that the behavior of the function in the limit of large $u_{x}$ is similar to that for $u_{x}$ near the maximum of the distribution. To validate this assumption, the distribution function is plotted against $u_{x}^{n}$ on a log-linear scale. The linear relationship of this plot ensures the validity of the obtained value of $n$. Figure 16 shows the distribution function plotted in a log scale along with the linear fit. No adjustable parameter is used in the fit. It is observed that the $k_{1}$, defined in Eq. (10) has a form $2 / \sqrt{\pi}\left(\sqrt{k_{2 m}}+\sqrt{k_{2 p}}\right)$, where $k_{2}=k_{2 p}$ for $u_{x}>u_{x m}$ and $k_{2}=k_{2 m}$ for $u_{x}<u_{x m}$. This indicates that there are two distinct velocity variances for the distribution function, one for velocities above $u_{x m}$ and the other for velocities below $u_{x m}$. The mean of this distribution is

$$
\left\langle u_{x}\right\rangle=u_{x m}+\frac{k_{2 p}-k_{2 m}}{\sqrt{\pi}\left(\sqrt{k_{2 m}}+\sqrt{k_{1 m}}\right)} .
$$

Figure 17 shows the distribution of the streamwise velocity and Fig. 18 the cross-stream velocity distribution on a loglinear scale for different values of $\epsilon$. The two distinct curves indicate that there exist two variances below and above the maximum value, as discussed above. Thus the simulation 


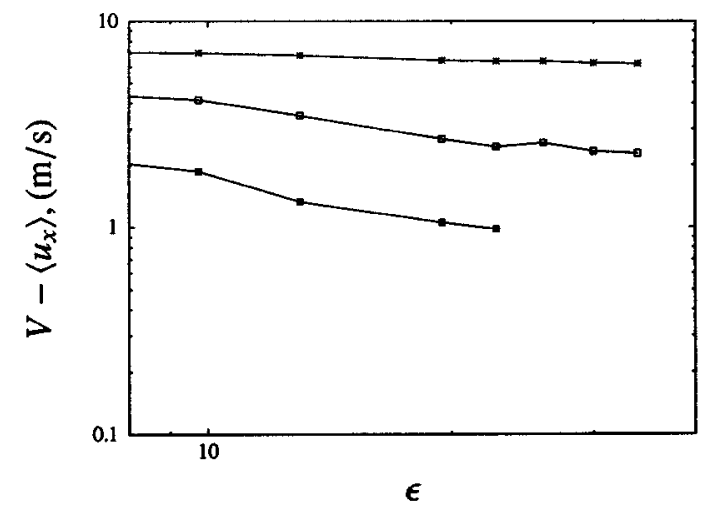

(a)

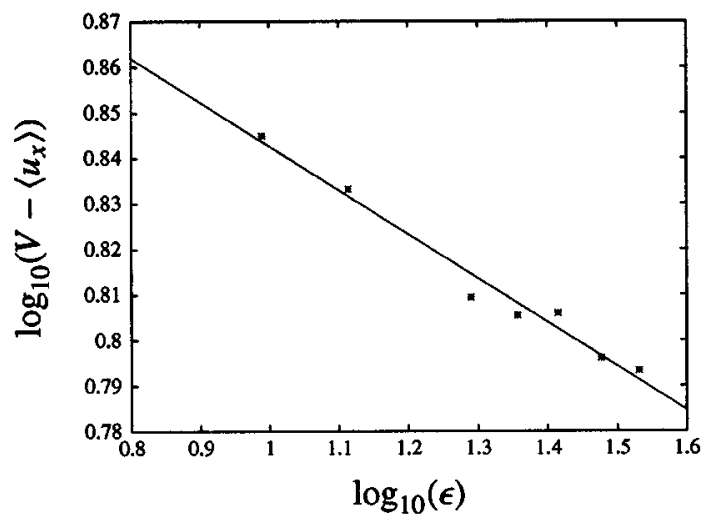

(b)

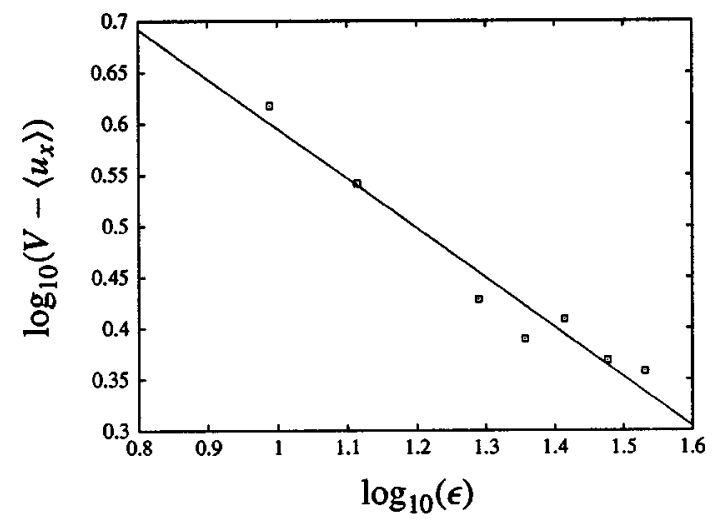

(c)

FIG. 22. Slip velocity at the wall for $e=0.99(*), e=0.9(\square)$, and $e=0.7$

results indicate that the nature of the velocity distribution near the wall differs drastically from that at the center of the channel for sheared inelastic granular materials at the low $\mathrm{Kn}$ limit.

Next, the macroscopic properties obtained from the simulations, such as the temperature, stress and heat flux, are examined. The temperature obtained from the simulation is compared with the temperature predicted in Ref. [3] for the steady, homogeneous flow between two material planes separated by a distance $L$ moving with a relative velocity of $V$. It was assumed that the density is a constant and the mean velocity varies linearly between these two plates, and obtained the granular temperature by a balance between the source of fluctuating energy due to the mean shear and the dissipation due to inelastic collisions. For smooth particles, the granular temperature is

$$
T=\frac{\mu \sigma^{2} \gamma^{2}}{4 \alpha(1-e)},
$$

where $\gamma$ is the strain rate and

$$
\begin{gathered}
\mu=\mu^{\prime}(1+v g(v) r)+\frac{1}{2} \alpha, \\
\alpha=\frac{8 m v^{2} g(v) r \sqrt{T}}{\pi^{3 / 2} \sigma},
\end{gathered}
$$

$$
\begin{gathered}
\mu^{\prime}=\frac{m \sqrt{T}}{B \sigma g(v) \sqrt{\pi}}[1+v g(v)(3 r-2) r], \\
r=\frac{(1+e)}{2}, \\
B=(5-3 r),
\end{gathered}
$$

and $g(v)$ is given in Ref. [28]. The temperature at the center of the channel is determined using the formulation, and is compared with the simulation results in Table I. The simulation results are in good agreement with the prediction of Ref. [3] at the center of the channel, but a spatial variation in the temperature and density is not predicted, since the flow is assumed to be homogeneous. This variation could be significant in the low Knudsen number limit, as observed in Fig. 19.

It is also observed that the second moments of the distribution $\left(\left\langle u_{x}^{2}\right\rangle\right.$ and $\left.\left\langle u_{z}^{2}\right\rangle\right)$ are anisotropic, (Fig. 20), and the difference in energy between the streamwise and cross-stream directions decreases as $\epsilon$ increases. It is useful to note that the difference in temperatures is about $20 \%$ at $e=0.9$, and as much as $50 \%$ at $e=0.7$. In the simulation results, there is a decrease in the temperature near the walls, due to the inelastic nature of the particle collisions with the walls. To examine this further, the local shear production of the energy and 
the local dissipation due to inelastic binary collisions are determined and listed in Table II. It is observed that the source due to the mean shear is larger than the dissipation due to binary collisions in all cases, and so there is a nonzero flux of energy towards the wall. The energy flux obtained from simulations is shown in Fig. 21. This figure indicates that the flux is positive near the top wall and negative near the bottom wall, indicating that there is a transfer of fluctuating energy from the particle to the wall.

The slip velocity at the wall has been calculated as the difference between the wall velocity and the mean velocity of system near wall, and is shown in Fig. 22. The slip velocity decreases as the coefficient of restitution decreases, and decreases as $\epsilon$ increases, though there is a significant slip velocity even at $\epsilon=0.5$.

\section{CONCLUSION}

The velocity distribution of a sheared granular material in a bounded channel is studied using an event driven simulation for a wide variation in the coefficients of restitution and the Knudsen number. In the limit of high Knudsen number, the distribution function for the streamwise velocity is bimodal, and the simulation results are found to be in good agreement with theoretical predictions in the limit $\epsilon \rightarrow 0$, where $\epsilon$, the ratio of the channel width and the distance between inter particle collisions, is inversely proportional to the Knudsen number. The scaling of the moments of the velocity distribution with $\epsilon$ are also in agreement with theoretical predictions, and the distribution function is nearly uniform across the channel. In the limit of low Knudsen number, the distribution function is observed to be close to Gaussian if the coefficient of restitution is close to 1 in the streamwise and cross-stream directions. However, if the coefficient of restitution is not close to 1, the streamwise distribution is a "composite Gaussian," which consists of two Gaussian distributions patched together at the location of the maximum velocity, with one variance for velocities above the maximum velocity and another for velocities below the maximum velocity.

The transition in the streamwise velocity distribution from the bimodal form for $\epsilon \ll 1$ to the composite Gaussian form for $\epsilon \gg 1$ occurs in a complicated fashion, and the distribution near the wall has three maxima at intermediate values of $\epsilon$, while the distribution at the center undergoes a transition from a bimodal to a unimodal form. The distribution function for the cross-stream velocity undergoes a transition from a delta function near zero velocity for $\epsilon \ll 1$ to a Gaussian for $\epsilon \gg 1$.

The predictions for the temperature of a sheared granular flow are accurate for determining the temperature at the center of the channel for high $\epsilon$, but do not capture the temperature and density variation near the wall of the channel even when $\epsilon$ is between 10 and 25. In particular, the flux of energy towards the wall of the channel due to the inelastic nature of the particle-wall collisions is not recovered in this limit. This, coupled with the complicated nature of the distribution function in the channel, indicates that the predictions of kinetic theories based on a Gaussian distribution may not accurately capture the dynamics of the flow when the coefficient of restitution is 0.9 or less even when the Knudsen number is between 10 and 25 .
[1] M. A. Hopkins and M. Y. Louge, Phys. Fluids A 3, 47 (1991).

[2] C. K. K. Lun, S. B. Savage, D. J. Jeffrey, and N. Chepurniy, J. Fluid Mech. 140, 223 (1984).

[3] J. T. Jenkins and M. W. Richman, Phys. Fluids 28, 3485 (1985).

[4] J. T. Jenkins and M. W. Richman, J. Fluid Mech. 171, 52 (1986).

[5] M. W. Richman and C. S. Chou, ZAMP 39, 885 (1998).

[6] P. C. Johnson and R. Jackson, J. Fluid Mech. 176, 67 (1987).

[7] T. N. Hanes, J. T. Jenkins, and M. W. Richman, J. Appl. Mech. 55, 969 (1988).

[8] C. K. K. Lun, Phys. Fluids 8, 2868 (1996).

[9] M. Babic, Phys. Fluids 9, 2486 (1997).

[10] M. Tij, M. Tahiri, J. M. Montanero, V. Garzo, A. Santos, and J. W. Dufty, J. Stat. Phys. 103, 1035 (2001).

[11] V. Kumaran, J. Fluid Mech. 340, 319 (1997).

[12] C. S. Campbell and C. E. Brennen, J. Fluid Mech. 151, 167 (1985).

[13] C. S. Campbell, J. Fluid Mech. 348, 85 (1997).
[14] C. S. Campbel and A. Gong, J. Fluid Mech. 164, 107 (1986).

[15] O. R. Walton and R. L. Braun, J. Rheol. 30, 949 (1986).

[16] C. S. Campbell and D. J. Wang, J. Fluid Mech. 244, 527 (1992).

[17] C. S. Campbell, J. Fluid Mech. 247, 111 (1993).

[18] C. S. Campbell, J. Fluid Mech. 203, 449 (1989).

[19] I. Goldhirsch and M. L. Tan, Phys. Fluids 8, 1752 (1996).

[20] M. L. Tan and I. Goldhirsch, Phys. Fluids 9, 856 (1997).

[21] M. Alam, J. T. Willits, B. O. Aranson, and S. Luding, Phys. Fluids 14, 4085 (2002).

[22] J. J. Brey, M. J. Ruiz-Montero and D. Cubero, Phys. Rev. E 59, 1256 (1999).

[23] F. Rouyer and N. Menon, Phys. Rev. Lett. 85, 3676 (2000).

[24] A. Kudrolli and J. Henry, Phys. Rev. E 62, R1489 (2000).

[25] W. Losert, D. G. W. Cooper, A. Kudrolli, and J. P. Gollub, Chaos 9, 682 (1999).

[26] T. van Noije and M. H. Ernst, Granular Matter 1, 57 (1998).

[27] J.-S. Olafsen and J. S. Urbach, Phys. Rev. E 60, R2468 (1999).

[28] L. Verlet and D. Levesque, Mol. Phys. 46, 969 (1982). 